Pacific Journal of Mathematics

TAME SUBSETS OF SPHERES IN $\boldsymbol{E}^{3}$ 


\title{
TAME SUBSETS OF SPHERES IN $E^{3}$
}

\author{
L. D. LOVELAND
}

Let $F$ be a closed subset of a 2-sphere $S$ in $E^{3}$. We define $F$ to be tame if $F$ lies on some tame 2-sphere in $E^{3}$. The sets $F$ and $S$ satisfy Property $(*, F, S)$ provided Bing's Side Approximation Theorem can be applied in such a way that the approximating 2-sphere $S^{\prime}$ misses $F$ (that is, $S \cap S^{\prime}$ lies in a finite collection of disjoint small disks in $S-F$ ). In this paper we show that Property $(*, F, S)$ implies that $F$ is tame by establishing a conjecture made by Gillman. Other properties which are equivalent to Property $(*, F, S)$ are also given.

If $F_{1}, F_{2}, \cdots, F_{n}$ is a finite collection of closed subsets of $S$ such that Property $\left(*, F_{i}, S\right)$ holds for each $i$, then Property $\left(*, \sum F_{i}, S\right)$ also holds. We use this result to show that if $S$ is locally tame modulo $\sum F_{i}$, then $S$ is tame.

Bing's Side Approximation Theorem [8, Theorem 16] can be stated as follows:

THEOREM 0. If $S$ is a 2-sphere in $E^{3}, V$ is a component of $E^{3}-S$, and $\varepsilon>0$, then there is a polyhedral 2-sphere $S^{\prime}$ containing a finite collection $D_{1}, D_{2}, \cdots, D_{n}$ of disjoint disks each of diameter less than $\varepsilon$, and there is a finite collection $E_{1}, E_{2}, \cdots, E_{r}$ of disjoint disks on $S$, each of diameter less than $\varepsilon$, such that

1. there is a homeomorphism of $S$ onto $S^{\prime}$ that moves no point as much as $\varepsilon$,

2. $S^{\prime}-\sum_{i=1}^{n} D_{i} \subset V$, and

3. $S \cap S^{\prime} \subset \sum_{i=1}^{r} E_{i}$.

If $F$ is a closed subset of the 2-sphere $S$ and $V$ is a component of $E^{3}-S$, we define Property $(*, F, V)$ to mean that Theorem 0 can be applied relative to $S$ and $V$ with the additional requirement that

4. $\left(\sum E_{i}\right) \cap F=\varnothing$.

Property $(*, F, S)$ is satisfied if Property $(*, F, V)$ holds for each component $V$ of $E^{3}-S$.

Gillman has already established that an are $A$ is tame if $A$ lies on a 2 -sphere $S$ and Property $(*, A, S)$ is satisfied; however, he comments that the "natural approach" to the problem requires a certain conjecture which he states and does not prove [13, p. 467]. Theorem 3 establishes this conjecture, and Theorem 6 shows that an arbitrary closed set $F$ on $S$ is tame if Property $(*, F, S)$ holds.

Hosay has announced two sufficient conditions for a closed subset 
$F$ of a 2-sphere $S$ to be tame [14]. In the statement of his results he requires that the set of diameters of the components of $F$ be bounded below by a positive number. Under this restriction on the size of the components of $F$ we show that each of Hosay's sufficient conditions is equivalent to Property $(*, F, S)$; hence, Hosay's result follows from Theorem 6 . For these results, see $\S 3$.

If $(*, F, S)$ is satisfied it follows from Theorem 1 that there is a nondegenerate continuum $M$ on $S$ such that $F \subset M$ and $(*, M, S)$ holds. Using Theorem 1, Theorem 8, and Hosay's result [14], we see that $(*, F, S)$ implies that $F$ is tame. Working independently, Ernest Milton also made this observation and established a theorem similar to Theorem 1. His work has not yet been published. As mentioned above, we do not depend on Hosay's result in this paper.

Theorems 17 and 18 are generalizations of Theorems 8.4 and 8.5 of [9]. We use these generalizations to show that the union $F$ of a finite collection of closed subsets $F_{1}, F_{2}, \cdots, F_{n}$ of a 2 -sphere $S$ satisfies $(*, F, S)$ provided Property $\left(*, F_{i}, S\right)$ holds for each $i$ (Theorem 21). It follows from this result and Theorem 15 that $S$ is tame if $S$ is locally tame modulo the union of a finite collection of closed subsets $F_{i}$ where $\left(*, F_{i}, S\right)$ holds for each $i$. We also use some of the results of Sections 3, 4, and 5 in another paper [16] where we give some conditions under which a 2 -sphere is tame in $E^{3}$.

We use the prefix in " $\varepsilon$-disk", " $\varepsilon$-set", etc., to imply that the point set in question has diameter less than $\varepsilon$. However, the prefix in "2-sphere" refers to the dimension of the sphere. It should be clear in which context the prefix is to be taken. The distance function in $E^{3}$ is denoted by " $\rho$ ". The symbol " $N(R, \varepsilon)$ ", where $\varepsilon>0$ and $R$ is a set, is synonymous with " $\varepsilon$-neighborhood of $R$ " and is defined as the set of all points that are within a distance $\varepsilon$ of some point of $R$. We indicate that a point set $R$ has diameter less than $\varepsilon$ by writing "diam $R<\varepsilon$ ". If $\left\{D_{i}\right\}$ is a countable sequence of disks such that lim diam $D_{i}=0$, we call $\left\{D_{i}\right\}$ a null sequence of disks. The closure of a set $R$ is denoted by $\mathrm{Cl}(R)$. If $S$ is a 2 -sphere we denote the bounded and unbounded components of $E^{3}-S$ by Int $S$ and Ext $S$, respectively. If $D$ is a disk we let Int $D=D-\operatorname{Bd} D$, where $\mathrm{Bd} D$ is the boundary of $D$.

Most of the definitions used here will be found in either [4] or [10]; however, we will review some of them briefly. A 2-sphere $S$ in $E^{3}$ is tame if there is a homeomorphism $h$ of $E^{3}$ onto itself such that $h(S)$ is polyhedral. The set $Y$ is locally simply connected at a point $p$ of $\mathrm{Cl}(Y)$ if for each neighborhood $N$ of $p$ there is an open set $U$ containing $p$ such that each map of a simple closed curve into $U \cap Y$ can be shrunk to a point in $N \cap Y$. A 2-sphere $S$ is said to be tame from a complementary domain $V$ of $S$ if $S+V$ is a 
3-manifold with boundary. The sphere $S$ is locally tame at a point $p$ from $V$ if $p$ is in $S$ and $p$ lies in a subset $U$ of $S+V$ such that $U$ is open relative to $S+V$ and $\mathrm{Cl}(U)$ is a topological cube.

2. $(*, F, S)$ implies that $\mathbf{F}$ is tame. We now give an alternative definition of Property $(*, F, S)$.

Property $(*, F, S)$. Let $F$ be a closed subset of a 2 -sphere $S$ in $E^{3}$. We say that Property $(*, F$, Ext $S)$ holds if and only if for each $\varepsilon>0$ there is a polyhedral 2-sphere $S^{\prime}$ containing a finite collection of disjoint $\varepsilon$-disks $D_{1}, D_{2}, \cdots, D_{n}$ and there is a finite collection of disjoint $\varepsilon$-disks $E_{1}, E_{2}, \cdots, E_{r}$ on $S$ such that

1. there is a homeomorphism of $S$ onto $S^{\prime}$ which moves no point as much as $\varepsilon$,

2. $S^{\prime}-\sum D_{i} \subset \operatorname{Ext} S$,

3. $S-\sum E_{i} \subset \operatorname{Int} S^{\prime}$, and

4. $F \cap\left(\sum E_{i}\right)=\varnothing$.

A similar definition is made for $(*, F$, Int $S)$, and we define $(*, F, S)$ to mean that both $(*, F$, Int $S)$ and $(*, F$, Ext $S)$ hold.

The property defined by $(*, F, S)$ in the introduction follows directly from this definition. The converse is also true as can be seen using the technique illustrated in [6, p. 585]. Since the two definitions of Property $(*, F, S)$ are equivalent we will use whichever definition seems appropriate.

Although we show in this section that Property $(*, F, S)$ implies that $F$ is tame, it is not true that $S$ is locally tame at points of $F$ if Property $(*, F, S)$ holds. To see this, let $F$ be an arc on the 2sphere $S$ described in [5]. Then $F$ is tame, so Property $(*, F, S)$ holds [13, Theorem 10]. Yet $S$ is not locally tame at any point.

THEOREM 1. If $\varepsilon>0$ and $F$ is a closed subset of a 2-sphere $S$ in $E^{3}$ such that $(*, F, S)$ is satisfied, then there is a continuum $M$ on $S$ and a null sequence $\left\{D_{i}\right\}$ of disjoint $\varepsilon$-disks on $S$ such that

1. $M=S-\sum \operatorname{Int} D_{i}$,

2. $(*, M, S)$ is satisfied, and

3. $F \subset M-\sum D_{i}=S-\sum D_{i}$.

Proof. Let $\varepsilon_{1}, \varepsilon_{2}, \varepsilon_{3}, \cdots$ be a sequence of positive numbers (subject to restrictions to be mentioned later), and let $S_{1}, S_{2}, S_{3}, \cdots$ be a sequence of polyhedral 2-spheres, where $S_{i}$ is obtained relative to $\varepsilon_{i}$ using Property $(*, F, S)$, such that for each $i$

(1) $S_{i}$ is homeomorphically within $\varepsilon_{i}$ of $S$,

(2) $S$ contains a finite collection of disjoint $\varepsilon_{i}$-disks $E_{i 1}, E_{i 2}, \cdots, E_{i n(i)}$ 
such that $\left(\sum_{j=1}^{n(i)} E_{i j}\right) \cap F=\varnothing$,

(3) $S_{i}$ contains a finite collection of disjoint $\varepsilon_{i}$-disks $D_{i 1}, D_{i 2}, \cdots, D_{i r(i)}$ such that

(a) $S_{i}-\sum_{i=1}^{r(i)} D_{i j} \subset \operatorname{Int} S$ and $S-\sum_{\jmath=1}^{n(t)} E_{i j} \subset \operatorname{Ext} S_{i}$ if $i$ is an odd integer, and

(b) $S_{i}-\sum_{j=1}^{r(i)} D_{i j} \subset \operatorname{Ext} S$ and $S-\sum_{j=1}^{n(i)} E_{i j} \subset \operatorname{Int} S_{i}$ if $i$ is an even integer,

We will impose restrictions on the $\varepsilon_{i}^{\text {'s }}$ to insure that $S$ $\sum_{i=1}^{\infty} \sum_{j=1}^{n(i)} E_{i j}$ contains a continuum $M$ which satisfies the requirements of Theorem 1. First we insist that $\varepsilon_{i}<\varepsilon / i$ for each $i$. We need an inductive procedure for defining the remaining restrictions to be imposed on the $\varepsilon_{\imath}$ 's. In this inductive procedure we also define an array of $\varepsilon$-disks

$$
\begin{aligned}
& G_{11} G_{12} \cdots G_{1 t(1)} \\
& G_{21} G_{22} \cdots G_{2 t(1)} \cdots G_{2 t(2)} \\
& G_{31} G_{32} \cdots G_{3 t(1)} \cdots G_{3 t(2)} \cdots G_{3 t(3)} \\
& \ldots
\end{aligned}
$$

where the $k^{\text {th }}$ row is a finite collection of disjoint $\varepsilon$-disks on $S$ such that $\sum_{i=1}^{n(k)} E_{k i} \subset \sum_{i=1}^{t(k)} \operatorname{Int} G_{k i} ; G_{k+1 i} \subset G_{k i}$ for $k=1,2, \cdots$ and $1 \leqq i \leqq$ $t(k)$ (that is, the columns are nested); and no $G_{k j}$ intersects $F$. We will also do the construction so that for each fixed row $k$, diam $G_{k j}<\varepsilon_{f}$ if $t(f-1)<j \leqq t(f)$ (where $t(0)=0$ and $1 \leqq f \leqq t(k)$ ).

Assuming we have defined such an array of disks, we let $A_{j}$ be the intersection of the $j^{\text {th }}$ column; that is $A_{j}=\bigcap_{i=1}^{\infty} G_{i j}$ (where we let $G_{i j}=S=\operatorname{Int} G_{i j} j>t(i)$ ). Since $A_{j}$ is the intersection of a nested collection of disks it follows that $A_{j}$ is a continuum. In fact we have defined a sequence of disjoint continua $A_{1}, A_{2}, A_{3}, \cdots$ on $S$ such that for each $i$

$$
\begin{gathered}
A_{i} \text { does not separate } S, \\
\operatorname{diam} A_{i}<\varepsilon, \\
\lim \operatorname{diam} A_{i}=0, \text { and } \\
A_{i} \cap F=\varnothing .
\end{gathered}
$$

In the inductive definition of the $G_{i j}$ 's we will insure that

(8) if $E_{m f}$ (where $m$ is a positive integer and $1 \leqq f \leqq n(m)$ ) lies in $G_{m k}$ (where $k$ is a positive integer such that $1 \leqq k \leqq t(m)$ ), then $E_{m f} \subset \bigcap_{i=1}^{\infty} G_{i k}=A_{k}$. 
Condition (8) implies that

$$
\sum_{i=1}^{\infty} \sum_{j=1}^{n(i)} E_{i j} \subset \sum_{i=1}^{\infty} A_{i} .
$$

Assuming that all these conditions are satisfied, let us show how the proof can be completed. From (6) the decomposition $G$ of $S$ whose only nondegenerate elements are the $A_{i}$ 's is upper semicontinuous. It follows from (4) and [18] that the decomposition space is a 2-sphere. Since there are only a countable number of nondegenerate elements in $G$, the image of these nondegenerate elements forms a countable point set in the decomposition space. Also from (7) this countable set fails to intersect the image of $F$ in the decomposition space. Using (5) and (6) we can find an infinite collection of disks in the decomposition space such that the pre-images of these disks form a null sequence of disjoint $\varepsilon$-disks $D_{1}, D_{2}, D_{3}, \cdots$ on $S$, where no $D_{i}$ intersects $F$ and $\sum_{l=1}^{\infty} A_{i} \subset \sum_{i=1}^{\infty} \operatorname{Int} D_{i}$. The continuum $M$ which is required in the conclusion of the statement of Theorem 1 is $S-\sum_{i=1}^{\infty} \operatorname{Int} D_{i}$.

Obviously $M$ contains $F$. Let $\alpha$ be a positive number, and let $V$ be a complementary domain of $S$. Since the $\varepsilon_{i}$ 's converge to zero we can find an odd integer $x$ and an even integer $y$ such that $\varepsilon_{x}$ and $\varepsilon_{y}$ are each less than $\alpha$. If $V=\operatorname{Int} S$, then $S_{x}$ will satisfy the conditions of $(*, M$, Int $S)$ relative to $\alpha$. If $V=\operatorname{Ext} S$, then $S_{y}$ will satisfy the conditions of $(*, M$, Ext $S)$ relative to $\alpha$. For example, consider $V=$ Int $S$. Then $S_{x}$ satisfies Conditions (1), (2), and (3a) as stated in the first paragraph of this proof. All we need to show is that $M$ does not intersect $\left(\sum_{j=1}^{n(x)} E_{x j}\right)$. But this follows immediately from (9) and the fact that the $A_{i}{ }^{\prime}$ s lie in the union of the interiors of the $D_{j}$ 's. Hence we have $(*, M, S)$.

Now let us show how the induction is carried out to define the $\varepsilon_{i}^{\prime}$ 's and the infinite array of $G_{i j}$ 's. We indicate the inductive procedure by illustrating the first three steps.

Step 1. No further restriction is placed on $\varepsilon_{1}$, so we let $S_{1}$ be a polyhedral 2-sphere satisfying (1), (2), and (3a). There is a finite collection of disjoint $\varepsilon_{1}$-disks $G_{11}, G_{12}, \cdots, G_{1 n(1)}$ so that $E_{1 i} \subset \operatorname{Int} G_{1 i}$ and $G_{1 i} \cap F=\varnothing$. For convenience in our inductive procedure we let $n(1)=t(1)$, and we let $H_{1 i}=E_{1 i}$ for $1 \leqq i \leqq t(1)$. We choose $\varepsilon_{2}$ to be less than $\rho\left(H_{1 i}, S-G_{1 i}\right)$ for $1 \leqq i \leqq t(1)$. Then $S_{2}$ is defined to satisfy (1), (2), and (3b).

Step 2. Let $K_{21}, K_{22}, \cdots, K_{2 t(1)}$ be the components of $\left(\sum_{i=1}^{t(1)} H_{1 i}+\right.$ $\left.\sum_{i=1}^{n(2)} E_{2 i}\right)$ such that $H_{1 i} \subset K_{2 i}$. From our choice of $\varepsilon_{2}, K_{2 i} \subset \operatorname{Int} G_{1 i}$. Let $H_{2 i}$ be $K_{2 i}$ plus the sum of all components of $S-K_{2 i}$ which lie in $G_{1 i}$, for $1 \leqq i \leqq t(1)$. Then 


$$
E_{1 i}=H_{1 i} \subset K_{2 i} \subset H_{2 i} \subset \operatorname{Int} G_{1 i}, \text { for } 1 \leqq i \leqq t(1) .
$$

For each $i$ such that $1 \leqq i \leqq t(1)$, we let $G_{2 i}$ be a disk in Int $G_{1 i}$ such that $G_{2 i} \cap\left(\sum_{i=1}^{t(1)} H_{1 i}+\sum_{i=1}^{n(2)} E_{2 i}\right)=H_{2 i} \subset \operatorname{Int} G_{2 i}$. Let $t(2)$ be a nonnegative integer so that exactly $t(2)-t(1)$ of the $E_{2 i}$ 's are not covered by $\sum_{1}^{t(1)} G_{2 i}$. We define $H_{2 t(1)+1}, \cdots, H_{2 t(2)}$ to be these uncovered $E_{2 i}$ 's. Now expand each $H_{2 i}(t(1)<i \leqq t(2))$ slightly to obtain a collection of disjoint $\varepsilon_{2}$-disks $G_{2 t(1)+1}, \cdots, G_{2 t(2)}$ covering $\sum_{t(1)+1}^{t(2)} H_{2 i}$ such that no $G_{2 i}$ intersects $F$. The $G_{2 i}$ 's are selected so that $G_{21}, G_{22}, \cdots, G_{2 t(2)}$ is a finite collection of disjoint $\varepsilon$-disks satisfying

$$
\begin{gathered}
\left(\sum_{i=1}^{n(1)} E_{1 i}+\sum_{i=1}^{n(2)} E_{2 i}\right) \subset \sum_{i=1}^{t(2)} \operatorname{Int} G_{2 i}, \\
H_{2 i} \subset \operatorname{Int} G_{2 i} \subset G_{2 i} \subset \operatorname{Int} G_{1 i}, \text { for } 1 \leqq i \leqq t(2), \\
\operatorname{diam} G_{2 i}<\varepsilon_{2} \text { for } t(1)<i \leqq t(2), \\
\left(\sum_{i=1}^{t(2)} G_{2 i}\right) \cap F=\varnothing, \text { and } \\
G_{2 i} \cap\left(\sum_{i=1}^{t(1)} H_{1 i}+\sum_{i=1}^{n(2)} E_{2 i}\right)=H_{2 i}, \text { for } 1 \leqq i \leqq t(2) .
\end{gathered}
$$

It follows from (12) that

$$
\operatorname{diam} G_{2 i}<\varepsilon_{1} \text { if } 1 \leqq i \leqq t(1) .
$$

Choose $\varepsilon_{3}$ less than $\rho\left(H_{2 i}, S-G_{2 i}\right)$ for $1 \leqq i \leqq t(2)$. Then $S_{3}$ is chosen relative to $\varepsilon_{3}$ so that $S_{3}$ satisfies (1), (2), and (3a).

Step 3. Let $K_{31}, K_{32}, \cdots, K_{3 t(2)}$ be the components of $\left(\sum_{i=1}^{t(2)} H_{2 i}+\right.$ $\left.\sum_{i=1}^{n(3)} E_{3 i}\right)$ such that $H_{2 i} \subset K_{3 i}$. From the definition of $\varepsilon_{3}, K_{3 i} \subset \operatorname{Int} G_{2 i}$. Define $H_{3 i}$ to be $K_{3 i}$ plus the sum of the components of $S-K_{3 i}$ which lie in Int $G_{2 i}(1 \leqq i \leqq t(2))$. Then

$$
H_{2 i} \subset K_{3 i} \subset H_{3 i} \subset \operatorname{Int} G_{2 i} \text { for } 1 \leqq i \leqq t(2) .
$$

For each $i$ such that $1 \leqq i \leqq t(2)$, we let $G_{3 i}$ be a disk in Int $G_{2 i}$ such that $G_{3 i} \cap\left(\sum_{i=1}^{t(2)} H_{2 i}+\sum_{i=1}^{n(3)} E_{3 i}\right)=H_{3 i} \subset \operatorname{Int} G_{3 i}$. Let $t(3)$ be a nonnegative integer so that there are exactly $t(3)-t(2)$ of the $E_{3 i}$ 's which are not covered by $\sum_{i=1}^{t(2)} G_{3 i}$, and let $H_{3 t(2)+1}, \cdots, H_{3 t(3)}$ be these uncovered $E_{3 i}$ 's. Now we expand each $H_{3 i}(t(2)<i \leqq t(3))$ so slightly that we obtain a collection of disjoint $\varepsilon_{3}$-disks $G_{3 t(2)+1}, \cdots, G_{3 t(3)}$ covering $\sum_{i=t(2)+1}^{t(3)} H_{3 i}$ such that no $G_{3 i}$ intersects $F$ and no $G_{3 i}(t(2)<i \leqq t(3))$ intersects a $G_{3 j}(1 \leqq j \leqq t(2))$. The collection $G_{31}, G_{32}, \cdots, G_{3 t(3)}$ can be selected to form a disjoint set of $\varepsilon$-disks satisfying

$$
\left(\sum_{i=1}^{n(1)} E_{1 i}+\sum_{i=1}^{n(2)} E_{2 i}+\sum_{i=1}^{n(3)} E_{3 i}\right) \subset \sum_{i=1}^{t(3)} \operatorname{Int} G_{3 i},
$$




$$
\begin{gathered}
H_{3 i} \subset \operatorname{Int} G_{3 i} \subset G_{13 i} \subset \operatorname{Int} G_{2 i} \text { for } 1 \leqq i \leqq t(3), \\
\operatorname{diam} G_{3 i}<\varepsilon_{3}, \text { for } t(2)<i \leqq t(3), \\
\left(\sum_{i=1}^{t(3)} G_{3 i}\right) \cap F=\varnothing, \text { and } \\
G_{3 i} \cap\left(\sum_{i=1}^{t(2)} H_{2 i}+\sum_{i=1}^{n(3)} E_{3 i}\right)=H_{3 i} \text { for } 1 \leqq i \leqq t(3) .
\end{gathered}
$$

From (13), (16), and (19) it follows that

$$
\begin{gathered}
\operatorname{diam} G_{3 i}<\varepsilon_{2} \text { if } t(1)<i \leqq t(2), \\
\operatorname{diam} G_{3 i}<\varepsilon_{1} \text { if } 1 \leqq i \leqq t(1)
\end{gathered}
$$

and

Choose $\varepsilon_{4}$ less than $\rho\left(H_{3 i}, S-G_{3 i}\right)$ for $1 \leqq i \leqq t(3)$, and let $S_{4}$ be defined to satisfy Conditions (1), (2), and (3b). Now we are ready to proceed with Step 4 where $\varepsilon_{5}$ is defined.

We assume that the inductive process is completed, so we have defined an array of $G_{i j}$ 's which satisfies the conditions required at the beginning of the proof. Perhaps we should elaborate on the reason that Condition (8) is satisfied. From the inductive procedure, as illustrated by Conditions (10) and (17), we see that $H_{1 j} \subset H_{2 j} \subset H_{3 j} \cdots$ for each $j$, provided we let $H_{i j}=\varnothing$ if $H_{i j}$ is not yet defined. Also from Conditions (12) and (19), carried through the inductive process, we have $\sum_{i=1}^{\infty} H_{i j} \subset \bigcap_{i=1}^{\infty} G_{i j}=A_{j}$. Suppose now that $E_{m f}$ lies in $G_{m k}$ (see (8)). Then the intersection of $G_{m k}$ with $\left(\sum_{i=1}^{t(m-1)} H_{m-1 i}+\sum_{l=1}^{n(m)} E_{m i}\right)$ is $H_{n z k}$ (see Condition (22)), so $E_{m f}$ lies in $H_{m k}$. Since $\sum_{i=1}^{\infty} H_{i k} \subset \bigcap_{\imath=1}^{\infty} G_{i k}$, Condition (8) holds.

The other conditions on the array of $G_{i j}$ 's are easily verified, so the proof is complete.

THEOREM 2. If $U$ is an open subset of a 2-sphere $S$ in $E^{3}$ and $F$ is a closed set in $U$ such that $(*, F, S)$ is satisfied, then there is a closed set $F^{\prime}$ containing $F$ such that

1. $F^{\prime}$ is a subset of $U$,

2. $\left(*, F^{\prime}, S\right)$ is satisfied, and

3. the set of diameters of the components of $F^{\prime}$ has a positive lower bound.

Proof. Let $V$ be an open set whose closure lies in $U$ such that $F$ lies in $V$. Choose a positive number $\varepsilon$ less than $\rho(F, \mathrm{Bd} V)$. From Theorem $1, F$ lies in a nondegenerate continuum $M$ in $S$ such that $(*, M, S)$ is satisfied. But $M$ might not lie in $U$ so we suppose that 


\section{$M$ intersects $\mathrm{Bd} U$.}

Let $Q$ be the set of all components of $M \cap \mathrm{Cl}(V)$ which intersect $F$. For each element $C$ in $Q$, $\operatorname{diam} C>\varepsilon$ since $C$ intersects both $F$ and $\operatorname{Bd} V$. Let $F^{\prime}$ be the sum of the elements of $Q$. Then $F^{\prime}$ satisfies Conditions 1,2, and 3 of Theorem 2. We need only show that $F^{\prime}$ is closed.

Suppose $F^{\prime}$ is not closed and let $p \in \mathrm{Cl}\left(F^{\prime}\right)-F^{\prime}$. Let $L$ be the component of $M \cap \mathrm{Cl}(V)$ which contains $p$. Then $L$ does not intersect $F$. There is a sequence of points $\left\{p_{i}\right\}$ converging to $p$ such that each $p_{i}$ lies in some $C_{i}$ in $Q$. It follows that $\lim \sup C_{i}$ is a subcontinuum $L^{\prime}$ of $L$ which contains $p$. Then $L^{\prime}$ fails to intersect $F$, so we let $N$ be an open set containing $L^{\prime}$ which does not intersect $F$. For each $i$, we can find a point $q_{i}$ in $C_{i}-N$, since otherwise some $C_{i}$ would lie in $N$ and consequently $N$ would intersect $F$. Then the set of $q_{i}$ 's has a limit point $q$ in $S-N$. The point $q$ must be in $L^{\prime}$ by the definition of $L^{\prime}$. Hence we have a contradiction. Then $F^{\prime}$ is closed.

The following lemma, which we state without proof, has been used by Gillman in the proof of Theorem 2 in [13].

Lemma 1. If $S$ is a 2-sphere in $E^{3}$ and $\varepsilon>0$, then there is a positive number $\delta$ so that if $f$ is a homeomorphism of $S$ which moves no point more than $\delta$, then any $\delta$-subset of $f(S)$ lies in a disk in $f(S)$ of diameter less than $\varepsilon$.

Theorem 3. If $U$ is an open subset of a 2-sphere $R$ in $E^{3}, F$ is a closed subset of $U$ such that $(*, F, R)$ is satisfied, and $S$ is a 2-sphere in $E^{3}$ containing $U$, then $(*, F, S)$ is also satisfied.

Proof. Let $\alpha$ be a positive number, and let $V$ be a complementary domain of $S$. We will construct a 2 -sphere $h(S)$ which satisfies the conditions $0:$ : $(*, F, V)$ relative to $\alpha$. The construction of $h(S)$ is similar to Bing's construction of $h(S)$ in his proof of Theorem 1 in [4], but the construction here could be considered simpler in the sense that we do not parallel his third approximation (the one which is obtained using Dehn's lemma). For convenience we assume $V=\operatorname{Int} S$, and Theorem 2 allows us to assume that the diameters of the components of $F$ are bounded below by $\alpha$. If $R=U$ the theorem is trivial, so we assume this is not the case.

Let $\varepsilon$ be a positive number such that each $\varepsilon$-subset of $S$ lies in a disk in $S$ of diameter less than $\alpha$. Then let $\varepsilon_{1}$ be a positive number satisfying the two conditions

$$
\begin{gathered}
3 \varepsilon_{1}<\rho(F, R-U), \text { and } \\
14 \varepsilon_{1}<\varepsilon .
\end{gathered}
$$


(a) A special cellular decomposition of $\boldsymbol{S}$. Let $T$ be a decomposition of $S$ into disks so that

(3) the diameter of each disk of $T$ is less than $\varepsilon_{1}$,

(4) the collection of disks of $T$ is the sum of three finite subcollections $A_{1}, A_{2}$, and $A_{3}$ such that no two elements of $A_{i}$ intersect $(i=1,2,3)$, and

(5) if $D$ and $D^{\prime}$ are two elements of $T$, then $\operatorname{Int} D$ and $\operatorname{Int} D^{\prime}$ do not intersect.

To see how to obtain such a decomposition, see [4, p. 296]. We let $K_{1}$ be the 1-skeleton of $T$; that is, $K_{1}$ is the sum of the boundaries of the disks of $T$.

(b) Pulling the disks of $T$ partially into Int $S$. Let $\delta$ be a positive number so small that the distance between two disks of $T$ without a common point is more than $\delta$. We also require that

$$
\delta<\varepsilon_{1}
$$

Using Lemma 1 , we let $\varepsilon_{2}$ be a positive number so that if $f$ is a homeomorphism of $R$ which moves points no more than $\varepsilon_{2}$, then each $\varepsilon_{2}$-subset of $f(R)$ lies in a $\delta / 6$-disk on $f(R)$. This, together with $(6)$, implies that

$$
\varepsilon_{2}<\delta / 6<\varepsilon_{1} / 6
$$

Let $S_{1}$ be a polyhedral 2-sphere and let $h_{1}$ be a homeomorphism of $S$ onto $S_{1}$ such that

(8) $h_{1}$ moves no point as much as $\varepsilon_{2}$,

(9) $S_{1}$ contains a finite collection of disjoint $\varepsilon_{z}$-disks $H_{1}, H_{2}, \cdots, H_{z}$ such that $S_{1}-\sum \operatorname{Int} H_{i} \subset \operatorname{Int} S$,

(10) $S$ contains a finite collection of disjoint $\varepsilon_{2}$-disks such that $S$ minus these disks lies in Ext $S_{1}$, and

$$
h_{1}\left(K_{1}\right) \subset S_{1}-\sum H_{i}
$$

For details on how to obtain $S_{1}$ and $h_{1}$, see [4, p. 296] and [8].

(c) The next approximation to elements of $\boldsymbol{T}$. For each disk $D$ in $T, h_{1}(D)$ is a first approximation to $D$. Notice that $h_{1}(D) \cap S$ lies in the sum of a finite collection of disjoint $\varepsilon_{2}$-disks in $h_{1}(\operatorname{Int} D)$ (this collection of disks is a subcollection of the disks $H_{i}$ of (9)), and 
$h_{1}(D)-\sum \operatorname{Int} H_{i}$ lies in Int $S$. It follows from (7) and (8) that

(12) $h_{1}(D)$ lies in a $\delta / 6$-neighborhood of $D$, for each disk $D \in T$.

We will now construct a second approximation $h_{2}(D)$ to each disk $D$ in $T$. Let $C$ be the set of all disks $D$ in $T$ such that $h_{1}(D)$ intersects $F$. For each disk $D$ in $T-C$, we choose a homeomorphism $h_{2}$ which agrees with $h_{1}$ on $D$; that is, for $D$ in $T-C$, the second approximation to $D$ is just $h_{1}(D)$. We must show how $h_{2}(D)$ is obtained if $D$ is in $C$.

Let $C^{\prime}$ be the set of all disks $D^{\prime}$ in $T$ such that there is a disk $D$ in $C$ which intersects $D^{\prime}$. Then for $D^{\prime}$ in $C^{\prime}$ it follows that there is a disk $D$ in $C$ such that $h_{1}(D)$ and $h_{1}\left(D^{\prime}\right)$ intersect. Notice that $C \subset C^{\prime}$, and $h_{2}$ is already defined on the disks in $C^{\prime}-C$.

Using (3), (7), and (8), it follows that

$$
\text { diam } h_{1}(D)<\varepsilon_{1}+2 \varepsilon_{2}<\varepsilon_{1}+\delta / 3<2 \varepsilon_{1}, \text { for each } D \in T \text {. }
$$

Consider a disk $D^{\prime}$ in $C^{\prime}$. There is a disk $D$ in $C$ such that $h_{1}(D)$ and $h_{1}\left(D^{\prime}\right)$ intersect. From $(7)$ we see that $2\left(\varepsilon_{1}+\delta / 3\right)$ is less than $3 \varepsilon_{1}$. Since $h_{1}(D)$ intersects $F$, we may use (13) to see that

$$
h_{1}\left(D^{\prime}\right) \text { lies in a } 3 \varepsilon_{1} \text {-neighborhood of } F \text {, for } D^{\prime} \text { in } C^{\prime} \text {. }
$$

From (14) and (1) it follows that

$$
h_{1}\left(D^{\prime}\right) \cap R \subset U, \text { for each disk } D^{\prime} \text { in } C^{\prime} \text {. }
$$

Then from (9) and (15) we have

$$
h_{1}\left(D^{\prime}\right)-\sum \operatorname{Int} H_{i} \subset E^{3}-R \text {, if } D^{\prime} \text { is a disk in } C^{\prime} \text {. }
$$

Thus we may choose a positive number $\varepsilon_{3}$ such that it is less than $\rho\left(h_{1}\left(D^{\prime}\right)-\sum \operatorname{Int} H_{i}, R\right)$ for each disk $D^{\prime}$ in $C^{\prime}$. We also require that

$$
\varepsilon_{3}<\varepsilon_{2} \text {. }
$$

Let $C_{1}^{\prime}$ be the set of all disks $D$ in $C^{\prime}$ such that $h_{1}(D)-$ $\sum$ Int $H_{i} \subset \operatorname{Ext} R$ and let $C_{1}=C \cap C_{1}^{\prime}$. Let $C_{2}^{\prime}$ be $C^{\prime}-C_{1}^{\prime}$, and let $C_{2}=C \cap C_{2}^{\prime}$. Notice that $C=C_{1}+C_{2}$, and $C^{\prime}=C_{1}^{\prime}+C_{2}^{\prime}$. If $D$ is in $C_{2}$, then $h_{1}(D)$ intersects $F$ and $h_{1}(D)-\sum \operatorname{Int} H_{i}$ lies in Int $R$. We will first show how to obtain $h_{2}(D)$ for the disks in $C_{1}$.

Using $\left(*, F\right.$, Ext $R$ ), we let $S^{\prime}$ be a polyhedral 2 -sphere and we let $h^{\prime}$ be a homeomorphism of $R$ onto $S^{\prime}$ such that

$h^{\prime}$ moves no point as much as $\varepsilon_{3}$,

(19) $S^{\prime}$ contains a finite collection of disjoint $\varepsilon_{3}$-disks such that $S^{\prime}$ minus these disks lies in Ext $R$, 
(20) $R$ contains a finite collection of disjoint $\varepsilon_{3}$-disks $Y_{1}, Y_{2}, \cdots, Y_{b}$ such that $R-\sum \operatorname{Int} Y_{i} \subset \operatorname{Int} S^{\prime}$, and

$$
\left(\sum Y_{i}\right) \cap F=\varnothing \text {. }
$$

Consider a disk $D$ in $C_{1}$. From our choice of $\varepsilon_{3}, S^{\prime} \cap h_{1}(D) \subset \sum \operatorname{Int} H_{i}$. We assume with no loss in generality that $h_{1}(D) \cap S^{\prime}$ consists of a finite number of disjoint simple closed curves, and we let $Q$ be the component of $h_{1}(D)-S^{\prime}$ which contains $\mathrm{Bd} h_{1}(D)$. The simple closed curves in $\operatorname{Cl}(Q) \cap S^{\prime}$ will be denoted by $J_{1}, J_{2}, \cdots, J_{r}$. Since each $J_{i}$ lies in some $H_{j}$ and diam $H_{j}<\varepsilon_{2}$, it follows that diam $J_{i}<\varepsilon_{2}$. Using (17), (18), and the definition of $\varepsilon_{2}$, we see that each $J_{i}$ bounds a disk $F_{i}$ in $S^{\prime}$ such that

$$
\operatorname{diam} F_{i}<\delta / 6 \text {. }
$$

From (18), the definition of $\varepsilon_{3}$, and $\left[15\right.$, p. 97] it follows that $h_{1}(D)-$ $\sum \operatorname{Int} H_{i} \subset \operatorname{Ext} S^{\prime}$ (this is true for all disks $D$ in $C_{1}^{\prime}$ ). We fill the holes in $Q$ with the $F_{i}^{\prime} s$, moving each $F_{i}$ slightly into Ext $S^{\prime}$ as we add it, to obtain a polyhedral disk $h_{2}(D)$ in $\operatorname{Ext} S^{\prime}$ [4, p. 297]. The $F_{i}^{\prime}$ s are moved to slightly that the new $F_{i}^{\prime}$ s also have diameter less than $\delta / 6$. Henceforth we use the symbol $F_{i}$ to denote the disk $F_{i}$ after it has been moved into Ext $S^{\prime}$. Notice that $h_{2}(D)$ does not intersect $F$ from (20) and (21). The homeomorphism $h_{2}$ is selected to agree with $h_{1}$ on $h_{1}^{-1}\left(h_{1}(D)-\sum \operatorname{Int} H_{i}\right)$. Thus we have obtained $h_{2}(D)$ for each disk $D$ in $C_{1}$.

For $D$ in $C_{2}$ we obtain $h_{2}(D)$ in just the same way as for the disks in $C_{1}$. The only difference is that we use $(*, F$, Int $R)$ to obtain a polyhedral 2-sphere $S$ ", homeomorphically within $\varepsilon_{3}$ of $R$, which "lies almost in Int $R$ and misses $F^{\prime \prime}$ (that is, $S^{\prime \prime}$ satisfies conditions similar to (18), (19), (20), and (21)). For each disk $D$ in $C_{2}, h_{1}(D)$ will lie "almost" in Int $S^{\prime \prime}$, so we can "pull $h_{1}(D)$ and $S$ " apart" just as we "pulled $h_{1}(D)$ and $S$ ' apart" in the preceding paragraph to obtain a polyhedral disk $h_{2}(D)$ in Int $S^{\prime \prime}$. Again $h_{2}$ is selected to agree with $h_{1}$ on $D-\sum h_{1}^{-1}\left(\operatorname{Int} H_{i}\right)$. Thus $h_{2}$ is defined for all disks $D$ in $T$ in such a way that

$$
h_{2}(D) \cap F=\varnothing, \text { for each disk } D \text { in } T \text {. }
$$

Now consider a disk $D$ in $C$. We will prove that the disks $F_{i}$, which replaced disks in $h_{1}(D)$ to form $h_{2}(D)$, do not intersect $S_{1}$ $\sum$ Int $H_{i}$. First we observe from the construction of $h_{2}(D)$ and (22) that

$$
h_{2}(D) \text { lies in a } \delta / 6 \text {-neighborhood of } h_{1}(D) \text {, for each } D \in T \text {. }
$$

From (12) and (24) we have 
$h_{2}(D)$ lies in a $\delta / 3$-neighborhood of $D$ for each $D \in T$.

If $D^{\prime}$ does not intersect $D$, it follows from (25) and the definition of $\delta$ that $h_{2}(D)$ and $h_{2}\left(D^{\prime}\right)$ fail to intersect. Thus if $D$ is in $C, F_{i}$ is a disk associated with $h_{2}(D)$, and $F_{i}^{\prime}$ intersects $h_{2}\left(D^{\prime}\right)$, then $D^{\prime}$ is in $C^{\prime}$. Consider a disk $D^{\prime}$ in $C^{\prime}$. It follows from the definition of $\varepsilon_{3}$ and (18) that $F_{i}$ does not intersect $h_{1}\left(D^{\prime}\right)-\sum \operatorname{Int} H_{i}$. Recalling that $h_{1}=h_{2}$ on $h_{1}^{-1}\left(h_{1}(D)-\sum \operatorname{Int} H_{i}\right)$, we see that no $F_{i}$ intersects $S_{1}-\sum \operatorname{Int} H_{i}$. Summarizing;

(26) If $h_{2}(D)$ and Int $h_{2}\left(D^{\prime}\right)$ intersect and $D \neq D^{\prime}$, then $D$ and $D^{\prime}$ are both in $C^{\prime}$. Furthermore if $D \neq D^{\prime}$, then $h_{2}\left(D^{\prime}\right)$ cannot intersect Int $h_{2}(D)$ in the set $\left(h_{1}(D)-\sum H_{i}\right)$.

(d) Untangling the $h_{2}(D)^{\prime}$ s. This section is the same as $\S(e)$ in $\left[4\right.$, p. 298] provided we substitute our $h_{2}(D)^{\prime}$ 's for the $h_{3}(D)^{\prime}$ 's in that paper. In order to be sure we have a 2 -sphere $h(S)$ after we use Bing's untangling process, we must be sure that if $D$ and $D^{\prime}$ are two elements of $T$ which do not intersect, then $h_{2}(D)$ and $h_{2}\left(D^{\prime}\right)$ also fail to intersect. However this follows directly from (25) and the definition of $\delta$.

Notice that from (26) we know the intersection of $h_{2}(D)$ with Int $h_{2}\left(D^{\prime}\right)$ does not intersect $S_{1}-\sum$ Int $H_{i}$ (unless $D$ is $D^{\prime}$ ). This means that the untangling process does not involve $S_{1}-\sum \operatorname{Int} H_{i}$, so we may choose $h$ equal to $h_{2}$ (and equal to $\left.h_{1}\right)$ on $h_{1}^{-1}\left(S_{1}-\sum \operatorname{Int} H_{i}\right)$. Thus $h(S)$ contains the set $S_{1}-\sum \operatorname{Int} H_{i}$, which is a 2 -sphere minus a finite collection of disjoint disks. To be sure that $h(S)$ is homeomorphically within $\alpha$ of $S$, we insure that the disks which are added to $S_{1}-\sum$ Int $H_{i}$ to form $h(S)$ are of small diameter. We show in $\S(e)$ that this has been accomplished. From (23) we see that the untangling can be done so that $h(S) \cap F=\varnothing$.

(e) The homeomorphism $\boldsymbol{h}$ moves no point as much as $\boldsymbol{\alpha}$. From (3), (25), and (6) it follows that $\operatorname{diam} h_{2}(D)<\operatorname{diam} D+2(\delta / 3)<2 \varepsilon_{1}$, for each disk $D$ in $T$. Since $h=h_{2}$ on each disk of $A_{1}$, we have

$$
\text { diam } h(D)<2 \varepsilon_{1} \text {, if } D \text { is a disk in } A_{1} \text {. }
$$

Let $D^{\prime}$ be a disk in $A_{2}$. To form $h\left(D^{\prime}\right)$ we added to $h_{2}\left(D^{\prime}\right)$ disjoint disks near $h(D)$ where $D$ is in $A_{1}$. Then from (27),

$$
\operatorname{diam} h\left(D^{\prime}\right)<\operatorname{diam} h_{2}\left(D^{\prime}\right)+2\left(2 \varepsilon_{1}\right)<6 \varepsilon_{1} \text {, if } D^{\prime} \in A_{2} .
$$

Let $D^{\prime \prime}$ be a disk in $A_{3}$. In forming $h\left(D^{\prime \prime}\right)$, we added to $h_{2}\left(D^{\prime \prime}\right)$ disks near $h(D)$ for $D$ in $A_{1}$ and disks near $h\left(D^{\prime}\right)$ for $D^{\prime}$ in $A_{2}$. Using (27) and (28) we see that each disk added to $h_{2}\left(D^{\prime \prime}\right)$ has diameter less than 
$6 \varepsilon_{1}$. Since each added disk intersects $h_{2}\left(D^{\prime \prime}\right)$, it follows that

(29) $h(D)$ lies in a $6 \varepsilon_{1}$-neighborhood of $h_{2}(D)$, for each $D \in T$, and

(30) $\operatorname{diam} h(D)<\operatorname{diam} h_{2}(D)+2\left(6 \varepsilon_{1}\right)<14 \varepsilon_{1}$, for each $D \in T$.

From (29) and (25), each point of $h(D)$ lies within a distance $6 \varepsilon_{1}$ of a point of $h_{2}(D)$ which lies within a distance $\delta / 3$ of a point of $D$. Hence no point is moved more than $6 \varepsilon_{1}+\delta / 3+\operatorname{diam} D<8 \varepsilon_{1}<\varepsilon<\alpha$; using (6), (3), and $\varepsilon<\alpha$.

(f) Property $(*, F$, Int $S)$ is satisfied. Since the homeomorphism $h$ was selected to agree with $h_{1}$ on the set $S-\sum h_{1}^{-1}\left(\operatorname{Int} H_{i}\right)$, each $\mathrm{Bd} H_{i}$ lies in some $h(D)$ and bounds a disk $D_{i}$ in that $h(D)$. From (30) and (2), diam $D_{i}<\varepsilon$. The collection of disjoint $\varepsilon$-disks $D_{1}, D_{2}, \cdots, D_{z}$ on $h(S)$ has the property that $h(S) \cap S \subset \sum \operatorname{Int} D_{i}$ (see Condition (9)). It follows from (9) and (11) that $h(S)-\sum D_{i} \subset \operatorname{Int} S$.

We must exhibit a finite collection of disjoint $\alpha$-disks on $S$ so that none of these disks intersect $F$ and so that $S \cap h(S)$ lies in the union of these disks. Since each component $Z$ of $h(S) \cap S$ lies in some $D_{j}$, diam $Z<\varepsilon$. From the definition of $\varepsilon, Z$ lies in an $\alpha$-disk on $S$. Assuming $2 \alpha$ is less than diam $S$ and recalling that $\alpha$ is a lower bound on the diameters of the components of $F$, we see that $F$ lies in the large component of $S-Z(Z \cap F=\varnothing$ because $h(S) \cap F=\varnothing)$, for each component $Z$ of $h(S) \cap S$. Following the procedure in the proof of Theorem 9 of [8], we obtain a finite collection of disjoint $\alpha$-disks $G_{1}, G_{2}, \cdots, G_{t}$ on $S$ such that $\left(\sum G_{i}\right) \cap F=\varnothing$ and $h(S) \cap S \subset\left(\sum G_{i}\right)$. Then $(*, F$, Int $S)$ is satisfied.

A similar procedure is used to establish $(*, F, \operatorname{Ext} S)$. Then it follows that $(*, F, S)$ is satisfied.

REMARK. The proofs of the next three theorems are modifications of Bing's proofs of Theorems 4, 5, and 1 in [6]. Consequently we do not carry out the details of the proofs, but merely outline the differences between his proofs and ours.

For what is meant by "a 2-sphere $S$ can be $\varepsilon$ approximated from Int $S$ (or Ext $S$ )", see [6]. We define " $H(A, B)<\varepsilon$ " to mean that $A$ and $B$ are homeomorphically within $\varepsilon$ of each other.

THEOREM 4. If $\varepsilon>0$ and $F$ is a closed subset of a 2-sphere $S$ in $E^{3}$ such that $(*, F$, Int $S)$ is satisfied, then there is a 2-sphere $S^{\prime}$ such that

1. $S^{\prime}$ is obtained from $S$ by removing a finite collection of disjoint $\varepsilon$-disks from $S-F$ and replacing them with $\varepsilon$-disks 
and

2. $S^{\prime}$ can be $\varepsilon$ approximated from Int $S^{\prime}$.

Proof. Because of the definition of $(*, F$, Int $S)$, we are able to accomplish in one paragraph all the essential elements in the first three paragraphs of Bing's proof of Theorem 4 in [6]. We know there is a polyhedral 2-sphere $S^{\prime \prime}$ containing a finite collection of disjoint $\varepsilon / 6$-disks $D_{1}, D_{2}, \cdots, D_{n}$ and there is a finite collection of disjoint $\varepsilon / 6$ disks $E_{1}, E_{2}, \cdots, E_{m}$ on $S$ such that $S^{\prime}-\sum D_{i} \subset \operatorname{Int} S, \quad S-$ $\sum E_{i} \subset \operatorname{Ext} S^{\prime \prime}$, no $E_{i}$ intersects $F$, and $H\left(S, S^{\prime \prime}\right)<\varepsilon / 6$. In fact since $F$ is closed, we may assume with no loss in generality that $S^{\prime \prime}-\sum \operatorname{Int} D_{i}$ lies in Int $S$ and $S-\sum \operatorname{Int} E_{i} \subset \operatorname{Ext} S^{\prime \prime}$.

The proof is completed by following Bing's paragraphs 4, 5, 6, and 7 in his proof of Theorem 4 in [6].

THEOREM 5. If $\varepsilon>0$ and $F$ is a closed subset of a 2-sphere $S$ in $E^{3}$ such that $(*, F, S)$ is satisfied, then there is a 2-sphere $S^{\prime}$ such that

1. $S^{\prime}$ is obtained by removing a finite number of disjoint $\varepsilon$-disks from $S-F$ and replacing them with $\varepsilon$-disks and

2. $S^{\prime}$ can be $\varepsilon$ approximated from each of its complementary domains.

Proof. The proof of Theorem 5 in [6] is followed here. We apply Theorem 4 to obtain a 2 -sphere $S_{1}$ such that

(1) $S_{1}$ is obtained by removing a finite collection $G$ of disjoint $\varepsilon / 4$ disks $E_{1}, E_{2}, \cdots, E_{m}$ from $S-F$ and replacing them with $\varepsilon / 4$ disks and

(2) there is a 2-sphere $S^{\prime \prime}$ in $\operatorname{Int} S_{1}$ such that $H\left(S_{1}, S^{\prime \prime}\right)<\varepsilon / 4$.

Let $\delta$ be a positive number subject to four restrictions to be mentioned later. Since $S-\sum E_{i}$ is an open subset of $S \cap S_{1}$ which contains $F$, it follows from Theorem 3 that $\left(*, F, S_{1}\right)$ is satisfied. This permits us to reapply Theorem 4, this time relative to Ext $S_{1}$ and $\delta$, to obtain a 2-sphere $S^{\prime}$ such that

(3) $S^{\prime}$ is obtained by removing a finite collection of disjoint $\delta$-disks $D_{1}, D_{2}, \cdots, D_{n}$ from $S_{1}-F$ and replacing them with $\delta$-disks and

(4) there is a polyhedral 2-sphere $S^{\prime \prime}$, in Ext $S^{\prime}$ such that $H\left(S^{\prime \prime}, S^{\prime}\right)<\delta$.

With suitable restrictions on $\delta, S^{\prime}$ is the required 2-sphere. 
Restrictions 1, 2, and 4 of the proof of Theorem 5 in [6] are also used here. We change Restriction 3 somewhat.

Restriction 3. We must identify a collection $H$ of disjoint $\varepsilon$-disks on $S$ which are to be replaced as mentioned in the first requirement in the conclusion of Theorem 5. For each $i(1 \leqq i \leqq m)$, let $E_{i}^{\prime}$ be an $\varepsilon / 3$-disk on $S$ such that $E_{i} \subset \operatorname{Int} E_{i}^{\prime}, E_{i}^{\prime} \cap F=\varnothing$, and $E_{\imath}^{\prime} \cap E_{j}^{\prime}=\varnothing$ if $i \neq j$. We choose $\delta$ so that $\delta<\rho\left(S-E_{i}^{\prime}, E_{i}\right)$ for each $i$. If we let $H_{i}$ be the component of $S \cap\left(\sum E_{i}+\sum D_{i}\right)$ which contains $E_{i}$ $(1 \leqq i \leqq m)$, it follows from our choice of $\delta$ that $H_{i}$ lies in Int $E_{i}^{\prime}$ for each $i$. Then we can find a simple closed curve $J_{i}$ in $E_{i}^{\prime}-\sum D_{i}$ which separates $S-E_{i}^{\prime}$ from $H_{i}$ in $S$. Let $L_{i}$ be the $\varepsilon / 3$-disk in $E_{i}^{\prime}$ which is bounded by $J_{i}$. Then $H_{i} \subset L_{i}$. Thus we have defined a finite collection of disjoint $\varepsilon / 3$-disks $L_{1}, L_{2}, \cdots, L_{m}$ such that for each $i$, $H_{i} \subset \operatorname{Int} L_{i}, L_{i} \cap F=\varnothing$, and $\left(\operatorname{Bd} L_{i}\right) \cap\left(\sum D_{j}\right)=\varnothing$.

Suppose the intersection with $S$ of some disk $D_{i}$ on $S_{1}$ is not covered by $\sum L_{i}$. Since $D_{i}$ fails to intersect any $L_{j}$, we know that $D_{i}$ lies in the intersection of $S$ and $S_{1}$. From Restriction 1 we know that $\delta<\varepsilon / 4$, so diam $D_{i}<\varepsilon / 4$. The collection $H$ is the set of all the $L_{i}^{\prime}$ s together with all the $D_{i}^{\prime}$ s which are not covered by the sum of the $L_{i}^{\prime}$ s.

THEOREM 6. If $\varepsilon>0$ and $F$ is a closed subset of a 2-sphere $S$ in $E^{3}$ such that $(*, F, S)$ is satisfied, then there is a continuum $M$ on $S$ and a null sequence $\left\{D_{i}\right\}$ of disjoint $\varepsilon$-disks on $S$ such that

1. $M=S-\sum \operatorname{Int} D_{i}$,

2. $(*, M, S)$ is satisfied,

3. $F \subset M-\sum D_{i}=S-\sum D_{i}$, and

4. M lies on a tame 2-sphere in $E^{3}$.

Proof. It follows from Theorem 1 that there is a continuum $M$ and a sequence $\left\{D_{i}\right\}$ of disks satisfying Conditions 1,2 , and 3 in the statement of Theorem 6. All we need to show is that there is a tame 2 -sphere in $E^{3}$ which contains $M$. This tame 2 -sphere will be constructed as the limit of a sequence of 2 -spheres $S_{1}, S_{2}, \cdots$, just as in the proof of Theorem 1 of [6]. In that proof Bing indicates three restrictions to be placed on the $S_{i}^{\prime}$ s to insure that

(1) $\lim S_{i}$ is a 2 -sphere,

(2) $\lim S_{i}$ is tame, and

(3) $\lim S_{i}$ shares a Sierpinski curve $X$ with $S$.

Our restrictions to insure that $\lim S_{i}$ is a tame 2-sphere are the same as those used by Bing in his Steps 1 and 2, provided we substitute our Theorem 5 wherever he uses his Theorem 5 . In the following paragraph we show that $\lim S_{i}$ shares $M$ with $S$. Of course 
(3) follows because of the construction of $M$.

Since $(*, M, S)$ is satisfied, we are able to use Condition 1 of Theorem 5 to obtain a 2-sphere $S_{1}$ such that $S_{1}$ is constructed by removing from $S$ a closed set $H_{1}$ which is the sum of a finite collection of disjoint $\varepsilon_{1}$-disks in $S-M$. Then $S-H_{1}$ is an open set in $S \cap S_{1}$ which contains $M$. Applying Theorem 3 we see that $\left(*, M, S_{1}\right)$ is satisfied.

Again we apply Condition 1 of Theorem 5, this time relative to $S_{1}$, to obtain a 2 -sphere $S_{2}$ such that $S_{2}$ is constructed by removing from $S_{1}$ a closed set $H_{2}$ which is the sum of a finite collection of disjoint $\varepsilon_{2}$-disks in $S_{1}-M$. Then $S_{1}-H_{2}$ is an open subset of $S_{1} \cap S_{2}$ which contains $M$. From Theorem 3 we have $\left(*, M, S_{2}\right)$ satisfied.

We continue this procedure so that $M \subset S_{i}$ for each $i$. This insures us that $M \subset \lim S_{i}$.

Remark. Gillman has shown that a 2 -sphere $S$ can be pierced by a tame arc at a point $p$ of $S$ if and only if $p$ lies in a tame arc on $S$ [13, Theorem 6]. It follows that $S$ can be pierced by a tame are at each point of the continuum $M$ identified in the conclusion of Theorem 6. Repeated application of Theorem 6 will thus establish that the set $Y$ of points of $S$ where $S$ cannot be pierced by a tame arc is a subset of a 0 -dimensional $G_{\delta}$ set. We state this result, without proof, as Theorem 7. Bing has already proven that $Y$ lies in a 0dimensional $G_{\delta}$ set [7, Theorem 5.2], and Gillman has shown that $Y$ is a 0 -dimensional $F_{\sigma}$ set [13, Theorem 11].

THeOREM 7. Each 2-sphere $S$ in $E^{3}$ contains a sequence $\left\{M_{i}\right\}$ of tame continua such that for each $i$

1. $M_{i} \subset M_{i+1}$,

2. $\left(*, M_{i}, S\right)$ holds,

3. $S-\sum M_{i}$ is a 0-dimensional $G_{\delta}$ set, and

4. $S$ can be pierced by a tame arc at each point of $\sum M_{i}$.

3. Conditions which are equivalent to $(*, F, S)$. In the following definitions we are considering $F$ to be a closed subset of a 2-sphere $S$ in $E^{3}$.

Property $(A, F, S)$. For each $\varepsilon>0$ there is a $\delta>0$ so that each $\delta$-simple closed curve in $E^{3}-S$ is homotopic to a constant (can be shrunk to a point) in an $\varepsilon$-subset of $E^{3}-F$.

Property $(B, F, S)$. There exist infinite sequences $\left\{S_{i}\right\}$ and $\left\{S_{i}^{\prime}\right\}$ of 2-spheres such that $S_{i}$ and $S_{i}^{\prime}$ are each homeomorphically within $1 / i$ of $S, F \subset \operatorname{Int} S_{i}$, and $F \subset \operatorname{Ext} S_{i}^{\prime}$. 
Hosay has announced that if the diameters of the components of $F$ have a positive lower bound and either of Properties $(A, F, S)$ or $\left(B, F^{\prime}, S\right)$ is satisfied, then $F$ lies on a tame 2-sphere in $E^{3}[14]$. It is our purpose to show that if the diameters of the components of $F$ have a positive lower bound, then not only are Properties $(A, F, S)$ and $(B, F, S)$ equivalent, but each is equivalent to Property $(*, F, S)$. The proof follows the pattern $(*, F, S) \Rightarrow(B, F, S) \Longrightarrow(A, F, S) \Longrightarrow(*, F, S)$. For convenience, we state the following two lemmas but do not include their proofs. A proof for Lemma 2 can be found in [10, Lemma 1], and Bing has stated Lemma 3 in [4, p. 294].

IsEMMa 2. If $D_{1}, D_{2}, \cdots, D_{n}$ is a finite collection of disjoint disks in $E^{3}$ and $f$ is a map of a disk $K$ into $E^{3}$ such that $f(\operatorname{Bd} K) \subset E^{3}-\sum D_{i}$, then there is a map $g$ of $K$ into $E^{3}$ such that

1. $g|\mathrm{Bd} K=f| \mathrm{Bd} K$,

2. $g(K) \subset f(K)+\sum \operatorname{Int} D_{i}$, and

3. $g(K)-\sum D_{i}$ is connected.

LEMma 3. If $F$ is a closed subset of a 2-sphere $S$ in $E^{3}$ such that for each point $p \in F$ and for each neighborhood $N$ of $p$ there is an open set $U$ such that $p \in U$ and each simple closed curve in $U-S$ can be shrunk to a point in $N-F$, then for each $\varepsilon>0$ there is a $\hat{\delta}>0$ such that each $\delta$-simple closed curve in $E^{3}-S$ can be shrunk to a point in an $\varepsilon$-subset of $E^{3}-F$.

THEOREM 8. If $F$ is a closed subset of a 2-sphere $S$ in $E^{3}$ such that $(*, F, S)$ is satisfied, then $(B, F, S)$ is also satisfied.

Proof. For each positive integer $i$ we must show the existence of 2 -spheres $S_{i}$ and $S_{i}^{\prime}$ such that each is homeomorphically within $1 / i$ of $S, F \subset \operatorname{Int} S_{i}$, and $F \subset \operatorname{Ext} S_{i}^{\prime}$. Using the definition of $(*, F$, Ext $S$ ) we obtain a polyhedral 2 -sphere $S_{i}$ containing a finite collection of disjoint $1 / i$-disks $D_{1}, D_{2}, \cdots, D_{m}$ and a finite collection of disjoint $1 / i$ disks $E_{1}, E_{2}, \cdots, E_{r}$ on $S$ such that $S$ and $S_{i}$ are homeomorphically within $1 / i, \quad S_{i}-\sum D_{j} \subset \operatorname{Ext} S, \quad S-\sum E_{j} \quad$ lies $\quad$ in Int $S_{i}, \quad$ and $\left(\sum E_{j}\right) \cap F=\varnothing$. It follows that $F$ lies in Int $S_{i}$.

The 2-sphere $S_{i}^{\prime}$ is obtained in the same manner using the definition of $(*, F$, Int $S)$.

TheOREM 9. If $F$ is a closed subset of a 2-sphere $S$ in $E^{3}$ such that $(B, F, S)$ is satisfied, then $(A, F, S)$ is also satisfied.

Proof. All that we need to show is that for each $p \in F$ and for 
each neighborhood $N$ of $p$ there is a neighborhood $U$ of $p$ such that each simple closed curve in $U-S$ is homotopic to a constant in $N-F$. The uniform condition in the definition of $(A, F, S)$ then follows from Lemma 3.

Let $p$ be a point of $F$, and let $N$ be a neighborhood of $p$ such that there are points of $S$ not in $N$. Let $K$ be a disk in $N \cap S$ such that $p$ is in Int $K$, and let $U$ be a neighborhood of $p$ such that $\mathrm{Cl}(U) \cap S \subset \operatorname{Int} K, \mathrm{Bd} U$ is a tame 2-sphere, and $\mathrm{Cl}(U) \subset N$. Let $J$ be a simple closed curve in $U-S$. Then $J$ is homotopic to a constant in $U$, so we let $f$ be a map of $K$ into $U$ such that $f$ restricted to $\mathrm{Bd} K$ is a homeomorphism onto $J$. For convenience we assume that $J$ is in Ext $S$.

Choose a positive number $\delta$ so that

(1) $\delta<\rho(J, S)$,

(2) $\delta<\rho(S-K, U)$, and

(3) $\delta<\rho(\mathrm{Bd} N, K)$.

Using $(B, F, S)$, we let $S^{\prime}$ be a 2 -sphere which is homeomorphically within $\delta$ of $S$ and such that $F \subset \operatorname{Int} S^{\prime}$. Let $h$ be a homeomorphism of $S$ onto $S^{\prime}$ such that

(4) $h$ moves no point as much as $\delta$.

Suppose that $S^{\prime}$ intersects $U$ in $S^{\prime}-h(K)$. Then there is a point $x$ in $S-K$ such that $h(x)$ is in $U$. This means that $\rho(x, h(x)) \geqq$ $\rho(S-K, U) \geqq \delta$, using (2). Since this contradicts (4) we have

(5) $U \cap S^{\prime} \subset h(K)$.

In a similar manner, using (3) and (4), it follows that

(6) $h(K) \subset N$.

Then from (1), (4) and [15, p. 97], we have

(7) $J \subset \operatorname{Ext} S^{\prime}$.

Condition (7) allows us to use Lemma 2 to obtain a map $g$ of $K$ into $E^{3}$ such that

(8) $g|\mathrm{Bd} K=f| \mathrm{Bd} K$,

(9) $g(K) \subset f(K)+\operatorname{Int} h(K)$, and

(10) $g(K)-h(K)$ is connected.

Suppose $g(K)$ is not a subset of $S^{\prime}+\operatorname{Ext} S^{\prime}$. Then the connected set $g(K)-h(K)$ intersects both complementary domains of $S^{\prime}$ and consequently intersects $S^{\prime}$. But from (5), (9), and the fact that $f(K) \subset U$, we see that this is impossible. Hence $g(K)$ lies in $S^{\prime}+\operatorname{Ext} S^{\prime}$, which means that $g(K) \cap F=\varnothing$. It follows directly from (6) and (9) that $g(K) \subset N$. Hence $g(K) \subset N-F$, and we have established Theorem 9 .

THEOREM 10. If $F$ is a closed subset of a 2-sphere $S$ in $E^{3}$ such that Property $(A, F, S)$ is satisfied and the diameters of the com- 
ponents of $F$ have a positive lower bound, then $(*, F, S)$ is satisfied.

Proof. The proof here is almost identical to Bing's proof that a 2-sphere in $E^{3}$ is tame if its complement is 1-ULC [4, Theorem 1]. Bing uses the 1-ULC condition to construct a 2 -sphere $h(S)$ which lies in Int $S$ and is homeomorphically close to $S$. Property $(A, F, S)$ permits us to use the same type construction to obtain 2-sphere $h(S)$ which satisfies all the requirements of Property $(*, F, S)$, although $h(S)$ need not lie in Int $S$. Bing has broken his proof into six sectionsa through e. We indicate how each of these sections can be changed to obtain a proof of Theorem 10 .

Let $\alpha>0$ and let $\varepsilon$ be a positive number so small that each $\varepsilon$ subset of $S$ lies in an $\alpha$-disk on $S$. With no loss in generality we assume that $\alpha$ is less than the diameter of each component of $F$. We choose a positive number $\varepsilon_{1}$ so that $14 \varepsilon_{1}$ is less than $\varepsilon$.

We will show that the conditions of Property $(*, F$, Int $S)$ are satisfied relative to the positive number $\alpha$. We follow exactly the same procedure that Bing followed in Sections a, b, and $c$ where he obtained a special cellular decomposition and applied the Side Approximation Theorem.

d. Third approximation to $\boldsymbol{D}$. It is here that the major change is made. (Notice that " $\mathrm{Bd} D$ " should be changed to " $h_{2}(\mathrm{Bd} D)$ " in the first paragraph of $\S d$ of Bing's paper). Everywhere Bing uses "Int $S$ " in his $\S d$ we substitute " $E^{3}-F$ ". The remainder of his $\S d$ is followed here except we allow the open set $U$, which contains the singular points of $g\left(h_{2}(D)\right)$, to intersect $S-F$, but we insist that $F \cap U=\varnothing$. This is possible since each $\mathrm{Bd} E_{i}^{\prime}$ is shrunk to a point missing $F$.

e. The fourth approximation to $D$. Bing's untangling procedure can be done so that no $h(D)$ intersects $F$ since each component of $h_{3}(S) \cap S$ lies in $U$.

f. Epsilontics. In the paragraph where Bing defines $\varepsilon_{4}$ we replace the second occurence of " $E^{3}-S$ " with " $E^{3}-F$ ", using $(A, F, S)$ in place of 1-ULC. The remainder of Bing's $\S f$ is followed here, but we must add a few comments to complete the proof.

Since $\delta_{2}<\rho\left(S, h_{1}(D)-\sum E_{i}\right)$, we know that $S^{\prime}$, as identified by Bing, intersects $h_{1}(D)$ only in $\sum$ Int $E_{i}$. Then $h_{2}$ may be selected to agree with $h_{1}$ on each $D-\sum h_{1}^{-1}\left(\operatorname{Int} E_{i}\right.$ ). Each point of $\mathrm{Bd} E_{i}^{\prime}$ (of $\S$ d) is within $\varepsilon_{4}$ of $S$ and $\mathrm{Bd} E_{i}^{\prime}$ is shrunk to a point in a $\delta_{2} / 2$-subset of $E^{3}-F$. This means that each $\mathrm{Bd} E_{i}^{\prime}$ is shrunk to a point in a set which is within $\varepsilon_{4}+\delta_{2} / 2$ of $S$. Since $\varepsilon_{4}<\delta_{2} / 2$, then $g\left(h_{2}(D)\right)$ has its 
singular points within $\delta_{2}$ of $S$. It follows from the definition of $\delta_{2}$ that $h_{3}$ may be chosen to agree with $h_{2}$ on $D-\sum h_{1}^{-1}\left(\operatorname{Int} E_{i}\right)$. Since $h_{1}(D)-\sum E_{i}$ is not involved in the untangling process described in $\S \mathrm{e}, h$ may also be chosen to agree with $h_{1}$ on $D-\sum h_{1}^{-1}\left(\operatorname{Int} E_{i}\right)$. It follows that each simple closed curve Bd $E_{i}$ lies in $h(D)$ and bounds a disk $R_{i}$ in $h(D)$. Bing has shown that $h(D)$ lies in a $6 \varepsilon_{1}$-neighborhood of $h_{3}(D)$ and that diam $h_{3}(D)$ is less than $2 \varepsilon_{1}$. Then

$$
\operatorname{diam} R_{i}<\operatorname{diam} h(D)<2 \varepsilon_{1}+2\left(6 \varepsilon_{1}\right)=14 \varepsilon_{1},
$$

which is less than $\varepsilon$. Each $h(D)$ has an associated collection of $R_{i}^{\prime}$ s, so we collect all these $R_{i}^{\prime}$ s together to form a finite collection of disjoint $\varepsilon$-disks $G_{1}, G_{2}, \cdots, G_{k}$ on $h(S)$ so that $h(S) \cap S \subset \sum \operatorname{Int} G_{i}$. Furthermore, since $h(D)-\sum \operatorname{Int} G_{i}=h_{1}(D)-\sum \operatorname{Int} E_{i}$ which lies in Int $S$, it follows that $h(S)-\sum G_{i} \subset \operatorname{Int} S$.

To show that $h(S)$ satisfies the conditions of $(*, F$, Int $S)$ relative to $\alpha$ we must exhibit a finite collection of disjoint $\alpha$-disks $H_{1}, H_{2}, \cdots$, $H_{r}$ on $S$ such that no $H_{i}$ intersects $F$ and $S \cap h(S) \subset \sum H_{i}$. Since each component $Z$ of $h(S) \cap S$ lies in an $\varepsilon$-disk $G_{i}$, it follows that $\operatorname{diam} Z<\varepsilon$. Then $Z$ lies in an $\alpha$-disk on $S$ (see the definition of $\varepsilon$ ). Since $\alpha$ is a lower bound on the diameters of the components of $F$ and no $Z$ intersects $F$, we may use the procedures in the proof of Theorem 9 of [8] to obtain disjoint $\alpha$-disks $\left\{H_{i}\right\}$ such that $H_{i} \cap F=$ $\varnothing$ and $h(S) \cap S \subset \sum H_{i}$.

Thus Property $(*, F$, Int $S)$ is satisfied. The proof that $(*, F$, Ext $S)$ holds is similar, so Property $(*, F, S)$ is satisfied.

REMARK. The requirement that $F$ be such that the diameters of its components are bounded below by a positive number cannot be removed from the hypothesis of Theorem 10. For let $S$ be the wild 2-sphere described by Fox and Artin [13] where the set of wild points of $S$ consists of a single point $p$. It is easy to see that Property $(B,\{p\}, S)$ is satisfied and that Property $(*,\{p\}, S)$ does not hold. However the following question apparently has not been answered. If $F$ is a closed subset of a 2 -sphere $S$ in $E^{3}$, Property $(B, F, S)$ is satisfied, and $F$ has no degenerate components, then will Property $(*, F, S)$ hold? Of course a similar question could be asked where Property $(A, F, S)$ replaces Property $(B, F, S)$.

We also note that if $F$ is a closed subset of a 2-sphere $S$ such that Property $(A, F, S)$ is satisfied, then $F$ need not lie in a nondegenerate subcontinuum $M$ of $S$ such that $(A, M, S)$ holds. To see this we use the same example as in the previous paragraph and let $F=\{p\}$. If a nondegenerate continuum $M$ exists such that $p \in M \subset S$ and $(A, M, S)$ holds, then $(*, M, S)$ also holds, but this is impossible. 
If Property $(A, F, S)$ holds for a closed subset $F$ of $S$ where $F$ has no degenerate components, then does $F$ lie in a nondegenerate continuum $M$ in $S$ such that $(A, M, S)$ holds?

The next two theorems are useful in the proofs of some theorems in [16], but they also lead to some interesting results in this paper. We define a simple closed curve in $E^{3}$ to be unknotted if it is the boundary of a tame disk in $E^{3}$.

THEOREM 11. Suppose $p$ is a point of a 2-sphere $S$ in $E^{3}, V$ is a complementary domain of $S, N$ is a neighborhood of $p$, and $K$ is $a$ disk. Then there is an open set $U$ containing $p$ such that for each closed subset $F$ of $S$ satisfying $(*, F, V)$ and for each map $f$ of $\mathrm{Bd} K$ into $U \cap V$ there is a finite collection of disjoint disks $H_{1}, H_{2}, \cdots, H_{t}$ in $(N-F) \cap S$ and there is a map $g$ of $K$ into $N$ such that $g \mid \mathrm{Bd} K=f$ and $g(K) \cap S \subset \sum \operatorname{Int} H_{i}$.

Proof. For convenience in notation we will assume $K$ is a disk on $S$ such that $p \in \operatorname{Int} K \subset K \subset N$. Let $K^{\prime}$ be a disk on $S$ such that $p \in \operatorname{Int} K^{\prime}$ and $K^{\prime} \subset \operatorname{Int} K$, and let $U$ be a neighborhood of $p$ such that $\mathrm{Cl}(U) \cap S \subset \operatorname{Int} K^{\prime}, \mathrm{Cl}(U) \subset N$, and $\mathrm{Bd} U$ is a tame 2-sphere. For convenience we assume $V=\operatorname{Ext} S$. Let $f$ be a map of $\mathrm{Bd} K$ into $U \cap V$. Since $\mathrm{Bd} U$ is tame we can extend $f$ to map all of $K$ into $U$.

Choose a positive number $\delta$ so that

(1) $\delta<\rho(f(\operatorname{Bd} K), S)$,

(2) $\delta<\rho\left(S-K, K^{\prime}\right)$,

(3) $\delta<\rho(\mathrm{Bd} N, K)$, and

(4) $\delta<\rho\left(S-K^{\prime}, U\right)$.

Now let $F$ be a closed subset of $S$ satisfying $(*, F$, Ext $S)$.

Using the definition of $(*, F$, Ext $S)$, we let $S^{\prime}$ be a polyhedral 2 -sphere containing a finite collection of disjoint $\delta$-disks $D_{1}, D_{2}, \cdots, D_{m}$ and let $E_{1}, E_{2}, \cdots, E_{r}$ be a finite collection of disjoint $\delta$-disks on $S$ such that

(5) There is a homeomorphism $h$ of $S$ onto $S^{\prime}$ such that $h$ moves no point as much as $\delta$,

(6) $S-\sum \operatorname{Int} E_{i} \subset \operatorname{Int} S^{\prime}$,

(7) $S^{\prime}-\sum D_{i} \subset$ Ext $S$, and

(8) $F \cap\left(\sum E_{i}\right)=\varnothing$.

Condition (6) does not come directly from the definition of $(*, F, S)$; however, from $(*, F, S)$ we have $S-\sum E_{i} \subset \operatorname{Int} S^{\prime}$. Then we can find a finite collection of disjoint $\delta$-disks $E_{1}^{\prime}, E_{2}^{\prime}, \cdots, E_{r}^{\prime}$ on $S$ such that $S-\sum \operatorname{Int} E_{i}^{\prime} \subset \operatorname{Int} S^{\prime}$ and no $E_{i}^{\prime}$ intersects $F$. Thus we may assume without loss in generality that Condition (6) holds.

It follows from (4) and (5) that

(9) $U \cap S^{\prime} \subset h\left(K^{\prime}\right)$, 
from (3) and (5) that

(10) $h\left(K^{\prime}\right) \subset N$,

and from (2) and (5) that

(11) $h\left(K^{\prime}\right) \cap S \subset K$.

From (1) and (5) we have $f(\operatorname{Bd} K) \subset E^{3}-h\left(K^{\prime}\right)$. This allows us to use Lemma 2 to obtain a map $g$ of $K$ into $E^{3}$ such that

(12) $g|\mathrm{Bd} K=f| \mathrm{Bd} K$,

(13) $g(K) \subset f(K)+\operatorname{Int} h\left(K^{\prime}\right)$, and

(14) $g(K)-h\left(K^{\prime}\right)$ is connected.

It follows from (13) and (10) that $g(K) \subset N$. Let $H_{1}, H_{2}, \cdots, H_{t}$ be the subcollection of the $E_{i}^{\prime}$ s such that each $H_{i}$ intersects $h\left(K^{\prime}\right)$ and $h\left(K^{\prime}\right) \cap S \subset \sum H_{i}$. Since each $H_{i}$ is a $\delta$-disk that intersects $h\left(K^{\prime}\right)$, it follows from (11), (3), and (8) that each $H_{i}$ lies in $(N-F) \cap S$.

Now we will show that $g(K) \cap S \subset \sum$ Int $H_{i}$. It follows from (12), (1), (5), and [15, p. 97] that $g(\mathrm{Bd} K)$ lies in Ext $S^{\prime}$. Suppose $g(K)$ intersects $S-\sum \operatorname{Int} H_{i}$. Then $g(K)$ intersects both components of $E^{3}-S^{\prime}$, by (6); hence, $g(K)-h\left(K^{\prime}\right)$ intersects each component of $E^{3}-S^{\prime}$. From (14), $g(K)-h\left(K^{\prime}\right)$ intersects $S^{\prime}$. Then from (13) and the fact that $f(K)$ lies in $U$, it follows that $g(K)-h\left(K^{\prime}\right)$ intersects $S^{\prime}$ in $U$. But this contradicts (9). Then $g(K) \cap S \subset \sum \operatorname{Int} H_{i}$.

Theorem 12. Suppose $p$ is a point of a 2-sphere $S$ in $E^{3}, V$ is a complementary domain of $S$, and $N$ is a neighborhood of $p$. Then there is an open set $U$ containing $p$ such that for each closed subset $F$ of $S$ which satisfies $(*, F, V)$ and for each unknotted simple closed curve $J$ in $U \cap V$ there is a finite collection of disjoint disks $H_{1}, H_{2}, \cdots, H_{t}$ in $(N-F) \cap S$ such that $J$ bounds a tame disk $D$ in $N$ and $D \cap S \subset \sum \operatorname{Int} H_{i}$.

Proof. The proof here is similar to the proof of Theorem 11. Let $K^{\prime}, K$, and $U$ be defined as in that proof, and let $J$ be an unknotted simple closed curve in $U \cap V$. Then $J$ bounds a tame disk $E$ in $U$. Now the proof here reads the same as the proof of Theorem $11 \mathrm{up}$ to and including Condition (11) if we identify $J$ with $f(\operatorname{Bd} K)$ and $E$ with $f(K)$.

We assume that $E$ is locally polyhedral at its interior points [2, Theorem 7] and that $E$ and $h\left(K^{\prime}\right)$ are in general position. Let $C$ be the component of $E-h\left(K^{\prime}\right)$ such that $J$ lies in $C$, and let $J_{1}, J_{2}, \cdots J_{n}$ be the components of $\mathrm{Cl}(C) \cap h\left(K^{\prime}\right)$. Since $E \cap S^{\prime} \subset h\left(K^{\prime}\right)$ and $J \subset \operatorname{Ext} S^{\prime}$, we know that each $J_{i}$ is a simple closed curve. Let $D_{1}, D_{2}, \cdots, D_{n}$ be the disks on $h\left(K^{\prime}\right)$ such that $\mathrm{Bd} D_{i}=J_{i}$ and assume that the $D_{i}^{\prime}$ s are ordered so that if $i<j$, then $D_{j}$ is not a subset of $D_{i}$. Now we add $D_{1}$ to $C$ and move $D_{1}$ slightly into Ext $S^{\prime}$. Next we add $D_{2}$ and move it into Ext $S^{\prime}$. We continue until all the $D_{i}^{\prime}$ s have 
been added to $C$ and adjusted, following a procedure described by Bing [4, p. 297]. Then we have a polyhedral disk $D$ in Ext $S^{\prime}$ such that $B d D=J$. The moving at each stage is done so that $D \subset N$ and $D \cap S$ lies in $\sum \operatorname{Int} E_{i}$.

The subcollection $H_{1}, H_{2}, \cdots, H_{t}$ of the $E_{i}^{\prime}$ s is chosen in the same way as in the proof of Theorem 11. Since $D$ lies in Ext $S^{\prime}$, it follows from (6) and (8) that $D \subset N-F$.

REMARK. Theorem 12 allows us to define another property which is equivalent to $(*, F, S)$ for certain closed sets $F$ on a sphere $S$.

Property $\left(A^{\prime}, F, S\right)$. Let $F$ be a closed subset of a 2 -sphere $S$ in $E^{3}$. If for each $\varepsilon>0$ there is a $\delta>0$ such that each unknotted simple closed curve of diameter less than $\delta$, which lies in $E^{3}-S$, bounds a tame $\varepsilon$-disk in $E^{3}-F$, then $F$ and $S$ are said to satisfy Property $\left(A^{\prime}, F, S\right)$.

If $(*, F, S)$ is satisfied, $p \in F$, and $N$ is a neighborhood of $p$, it follows from Theorem 12 that there is a neighborhood $U$ of $p$ such that each unknotted simple closed curve in $U-S$ bounds a tame disk in $N-F$. Thus we can apply an argurment similar to the proof of Lemma 3 to show that $(*, F, S)$ implies $\left(A^{\prime}, F, S\right)$.

The converse is also true provided the diameters of the components of $F$ have a positive lower bound. To see this we observe that in the proof of Theorem 10 all we needed was to have certain small unknotted simple closed curves in $E^{3}-S$ bound small tame disks missing $F$. Thus the following theorem holds.

THEOREM 13. If the diameters of the components of the closed subset $F$ of a 2-sphere $S$ in $E^{3}$ have a positive lower bound, then $\left(A^{\prime}, F, S\right)$ and $(*, F, S)$ are equivalent.

THEOREM 14. If $S$ is a 2-sphere in $E^{3}, V$ is a component of $E^{3}-S, F$ is a closed subset of $S,(*, F, V)$ holds, and $W$ is an open subset of $S$ such that $V$ is locally simply connected at each point of $W-F$ (or equivalently, $S$ is locally tame from $V$ at each point of $W-F)$, then $S$ is locally tame from $V$ at each point of $W$.

Proof. Let $p \in F \cap W$, and let $N$ be a neighborhood of $p$ such that $N \cap S \subset W$. Let $U$ be an open set containing $p$ such that $U$ satisfies the conditions of Theorem 11 relative to $V, N$, and $p$. Let $f$ be a map of the boundary of a disk $K$ into $U \cap V$.

Using the properties of $U$ guaranteed by Theorem 11, we let $H_{1}, H_{2}, \cdots, H_{t}$ be a finite collection of disjoint disks in $(N-F) \cap S$ 
and we let $g$ be a map of $K$ into $N$ so that $g \mid \mathrm{Bd} K=f$ and $g(K) \cap S \subset \sum \operatorname{Int} H_{i}$. Let $X$ be an arc from a point $b \in f(\operatorname{Bd} K)$ to a point $q$ in $S-\sum H_{i}$ such that $X-q$ lies in $V$. For each $i(1 \leqq i \leqq t)$ there is a disk $H_{i}^{\prime}$ such that $\mathrm{Bd} H_{i}^{\prime}=\mathrm{Bd} H_{i}$, Int $H_{i}^{\prime} \subset V \cap N$, and $g(\operatorname{Bd} K)+X$ lies in $\operatorname{Ext}\left(H_{i}+H_{i}^{\prime}\right)$. This is because $S$ is tame from $V$ at each point of each $H_{i}[1 ; 17]$.

Using Lemma 2 we obtain a map $h$ of $K$ into $E^{3}$ such that $h \mid \operatorname{Bd} K=f, h(K) \subset g(K)+\sum \operatorname{Int} H_{i}^{\prime}$, and $h(K)-\sum H_{i}^{\prime}$ is connected. Obviously $h(K) \subset N$. Since $h(K)$ is connected and $h(\mathrm{Bd} K) \subset V$, it will follow that $h(K) \subset V$ if we show that $h(K) \cap S=\varnothing$. To show this we use the fact that $h(K)-\sum H_{i}^{\prime}$ is arcwise connected.

Suppose $h(K)$ intersects $S$. Let $Y$ be an arc in $h(K)-\sum H_{i}^{\prime}$ from the point $b$ to a point $d$ in $S$ such that $(Y-d) \subset V$. Since $g(K) \cap S \subset \sum$ Int $H_{i}$ and $h(K) \subset g(K)+\sum$ Int $H_{i}^{\prime}$, it follows that $d \in \operatorname{Int} H_{j}$ for some $j(1 \leqq j \leqq t)$. Let $Z$ be an arc from the point $q$ to $d$ such that $Z-(d+q) \subset E^{3}-(S+V)$. Then $X+Y+Z$ contains a simple closed curve $L$ which links $\mathrm{Bd} H_{j}$ [7, Theorem 3.3], and it follows from Theorem 10 of [2] that $L$ links $\mathrm{Bd} H_{j}^{\prime}$. Therefore $L$ intersects $H_{j}^{\prime}$. But this is a contradiction since $X+Y+Z$ does not intersect $H_{j}^{\prime}$.

Thus $h(K) \subset N \cap V$, so $V$ is locally simply connected at each point $p$ of $W \cap F$. Then, from the hypothesis, $V$ is locally simply connected at each point of $W$. Using Theorems 1 and 2 of [10], it follows that $S$ is locally tame from $V$ at each point of $W$.

Theorem 15. If a 2-sphere $S$ in $E^{3}$ is locally tame modulo a closed subset $F$ which satisfies $(*, F, S)$, then $S$ is tame.

Proof. Using $W=S$ in Theorem 14, we have the result that $S$ is locally tame from each of its complementary domains. Then $S$ is tame from each such domain; hence $S$ is tame.

REMARK. It follows from Theorem 15 that $F$ cannot contain an isolated wild point of $S$ if $(*, F, S)$ is satisfied. Also the set $W$ of wild points of a 2 -sphere $S$ fails to satisfy $(*, W, S)$, since $W$ is closed.

4. Property $(*, F)$ and its relation to $(*, F, S)$. We now define Property $(*, F)$. If a closed subset $F$ of a 2 -sphere $S$ in $E^{3}$ has no degenerate components, we suspect that Property $(*, F)$ is satisfied if and only if Property $(*, F, S)$ holds. However Theorem 16 is as close as we have come to establishing this equivalence.

Property $(*, F)$. A closed set $F$ has Property $(*, F)$ if and only if it satisfies each of the following conditions: 
1. $\quad F$ is a subset of some 2 -sphere in $E^{3}$.

2. If $S$ is a 2 -sphere in $E^{3}$ such that $S$ contains $F$ and $S$ is locally tame modulo $F$, then $S$ is tame.

If each of the following sets lies on some 2-sphere in $E^{3}$, then each is an example of a set $F$ satisfying property $(*, F)$ : (1) a tame disk [2; 11], (2) a tame finite graph [11, Corollary 1], (3) a tame Sierpinski curve [9, Theorem 8.2], and (4) a set which is the union of a finite number of tame finite graphs and tame Sierpinski curves [9, Theorem 8.4]. If $F$ consists of a single point, it is easy to see that $F$ does not satisfy $(*, F)$ (see the example of a 2-sphere which is wild at a single point, as given in [12]). This raises a question which is related to one asked in [13, p. 464]. Does a closed set $F$ satisfy $(*, F)$ if $F$ lies in a tame 2-sphere and $F$ has no degenerate components? We do not answer this question.

THEOREM 16. A closed subset $F$ of a 2-sphere satisfies $(*, F)$ if and only if $(*, F, S)$ is satisfied relative to each 2 -sphere $S$ containing $F$.

Proof. Suppose $F$ has Property $(*, F, S)$ relative to each 2-sphere $S$ containing $F$. We want to show that $(*, F)$ holds, so we let $S^{\prime}$ be a 2-sphere such that $S^{\prime}$ contains $F$ and $S^{\prime}$ is locally tame modulo $F$. We apply Theorem 15 to see that $S^{\prime}$ is tame. As suggested by Gillman, the other half of Theorem 16 is proved using the techniques in the proof of Theorem 2 in [13].

THEOREM 17. If $S^{*}$ is a 2-sphere in $E^{3}$ containing a finite collection of closed sets $F_{1}, F_{2}, \cdots, F_{n}$ such that each $F_{i}$ satisfies $\left(*, F_{i}^{\prime}\right)$, then $\left(*, \sum F_{i}\right)$ also holds.

Proof. Let $S$ be a 2-sphere containing $\sum F_{i}$ such that $S$ is locally tame modulo $\sum F_{i}$. We will complete the proof by showing that $S$ is tame. First we observe from Theorem 16 that $\left(*, F_{i}, S\right)$ holds for each $i$. Now an application of Theorem 14 shows that $S$ is locally tame from Ext $S$ at each point of the open set $U_{1}=S-\sum_{i=2}^{n} F_{i}$. Another application of Theorem 14 shows that $S$ is locally tame from Ext $S$ at each point of the open set $U_{2}=S-\sum_{i=3}^{n} F_{i}$. We continue applying Theorem 14 until we have $S$ locally tame from Ext $S$ at each point of $U_{n}=S$. Then $S$ is tame from Ext $S$. A similar argument shows that $S$ is tame from Int $S$. Hence $S$ is tame.

THEOREM 18. If $S$ is a 2-sphere in $E^{3} ; F_{1}, F_{2}, \cdots, F_{n}$ is a finite collection of closed subsets of $S$ such that $\left(*, F_{i}\right)$ holds for each $i$; 
and $U$ is an open subset of $S$ such that $S$ is locally tame at each point of $U-\left(\sum F_{i}\right)$, then $S$ is locally tame at each point of $U$.

Proof. Although a proof similar to the proof of Theorem 17 can also be given here, we give an alternative method. We apply Theorem 17 to see that $\left(*, \sum F_{i}\right)$ holds; then we use Theorem 16 to see that $\left(*, \sum F_{i}, S\right)$ is true. Now two applications of Theorem 14 will show that $S$ is locally tame from each of its complementary domains at each point of $U$. Thus $S$ is locally tame at each point of $U$.

REMARK. It is known that if $F$ lies on some 2-sphere in $E^{3}$ and $F$ is either a tame finite graph or a tame Sierpinski curve, then $(*, F)$ is satisfied $[11 ; 9]$. Theorem 19 shows the existence of a continuum $M$ satisfying $(*, M)$ such that $M$ is not a finite union of tame finite graphs and tame Sierpinski curves. It follows that Theorems 17 and 18 are generalizations of Theorems 8.4 and 8.5, respectively, in [9]. It also follows from Theorem 19 that the continuum $M$ in the conclusion of Theorem 6 satisfies $(*, M)$.

Theorem 19. If $M$ is a tame continuum on a 2-sphere $S$ in $E^{3}$ such that $M$ is obtained by removing from $S$ the interiors of a null sequence of disjoint disks on $S$, then $(*, M)$ is satisfied.

Proof. Let $M=S-\sum \operatorname{Int} D_{i}$, where $\left\{D_{i}\right\}$ is a null sequence of disjoint disks on $S$. Suppose $S^{\prime}$ is a 2-sphere containing $M$ such that $S^{\prime}$ is locally tame $\bmod M$. We will show that $S^{\prime}$ is tame.

Let $E_{i}$ be the disk on $S^{\prime}$ such that $\mathrm{Bd} E_{i}=\mathrm{Bd} D_{i}$, for each $i$. It is easily seen that $\left\{E_{i}\right\}$ must be a null sequence.

Let $G=\left\{H_{1}, H_{2}, \cdots\right\}$, be an infinite collection of disjoint disks of $S^{\prime}$ such that $\lim \operatorname{diam} H_{i}=0$, each $E_{i}$ is in $G$, and $\sum H_{i}$ is dense in $S^{\prime}$. Then $S^{\prime}-\sum \operatorname{Int} H_{i}$ is a Sierpinski curve $K$ [20], and since $K \subset M$, it follows that $K$ is tame. Furthermore, since $S^{\prime}$ is locally tame at each point of $\sum \operatorname{Int} H_{i}$, it follows that $S^{\prime}$ is locally tame modulo $K$. Since $(*, K)$ is satisfied [9], then $S^{\prime}$ is tame.

Theorem 20. If $S$ is a 2-sphere in $E^{3},\left\{D_{i}\right\}$ is a null sequence of disjoint disks on $S$, and $M=S-\sum \operatorname{Int} D_{i}$, then the following statements are equivalent:

1. $M$ is tame.

2. $(A, M, S)$ is satisfied.

3. $(*, M, S)$ is satisfied.

4. $(*, M)$ is satisfied.

5. $(B, M, S)$ is satisfied.

6. $\left(A^{\prime}, M, S\right)$ is satisfied. 
Proof. Since $M$ is a nondegenerate continuum, we have already proven that Statements 2, 3, 5, and 6 are equivalent (see Theorems $8,9,10$, and 13). Bing's proof of Theorem 8.1 in [9] also shows that Statement 1 implies 2 . Since 2 implies 3 , the proof will be completed if we show that 3 implies 4 and 4 implies 1 . If 3 is satisfied it follows from Theorem 6 that 1 is true. Then 4 follows from Theorem 19. Thus 3 implies 4 . Section 8 of [8] shows the existence of a 2sphere $S^{\prime}$ containing $M$ such that $S^{\prime}$ is locally tame modulo $M$. Then 4 implies 1 because $S^{\prime}$ is tame if 4 is satisfied.

REMARK. Statements 1 through 6 of Theorem 20 characterize tame Sierpinski curves on 2 -spheres in $E^{3}$. This follows because if $K$ is a Sierpinski curve on $S$, then there is a null sequence of disjoint disks $D_{1}, D_{2}, \cdots$, on $S$ such that $K=S-\sum \operatorname{Int} D_{i}$ [19].

5. Finite sums of sets $F$ satisfying $(*, F, S)$. The following theorem is used in [16].

TheOREM 21. If $F_{1}, F_{2}, \cdots, F_{n}$ is a finite collection of closed subsets of a 2-sphere $S$ in $E^{3}$ such that $\left(*, F_{i}, S\right)$ is satisfied for each $i$, then $\left(*, \sum F_{i}, S\right)$ also holds.

Proof. We use Theorem 6 to obtain a finite collection of tame continua $M_{1}, M_{2}, \cdots, M_{n}$ on $S$ such that, for each $i,\left(*, M_{i}, S\right)$ is satisfied, $F_{i} \subset M_{i}$, and $M_{i}$ is obtained by removing from $S$ the interiors of a null sequence of disjoint disks on $S$. From Theorem 19 we see that $\left(*, M_{i}\right)$ is satisfied for each $i$. Then it follows from Theorem 17 that $\left(*, \sum M_{i}\right)$ holds. Now we use Theorem 16 to see that $\left(*, \sum M_{i}, S\right)$ is satisfied, and $\left(*, \sum F_{i}, S\right)$ follows because $\sum F_{i} \subset \sum M_{i}$.

REMARK. We note that Theorem 21 cannot be extended to the case where the $F_{i}^{\prime}$ s form an infinite collection of closed subsets of $S$. To see this, let $S$ be a wild 2-sphere in $E^{3}$ and for each $i$ let $F_{i}$ be a continuum $M_{i}$ as in the statement of Theorem 7 . Then $\left(*, M_{i}, S\right)$ holds for each $i$ and $S-\sum M_{i}$ is a 0 -dimensional set. Suppose $\left(*, \Sigma M_{i}, S\right)$ holds. Since $S-\sum M_{i}$ contains no disk it follows that $S$ can be homeomorphically approximated in each of its complementary domains. This is a contradiction since under these conditions $S$ is tame [3, Theorem 2.2]. Is $\left(*, \sum F_{i}, S\right\}$ satisfied if $\left\{F_{i}\right\}$ is an infinite collection of closed subsets of $S$ such that $\sum F_{i}$ is closed and $\left(*, F_{i}, S\right)$ holds for each $i$ ?

THEOREM 22. If $F_{1}, F_{2}, \cdots, F_{n}$ is a finite collection of closed subsets of a 2-sphere $S$ in $E^{3}$ such that for each $i$ 
1. either $\left(A, F_{i}, S\right)$ or $\left(B, F_{i}, S\right)$ is satisfied, and

2. the diameters of the components of $F_{i}$ have a positive lower bound,

then $\left(A, \sum F_{i}, S\right)$ and $\left(B, \sum F_{i}, S\right)$ are satisfied.

Proof. Because $\left(*, F_{i}, S\right),\left(A, F_{i}, S\right)$, and $\left(B, F_{i}, S\right)$ are equivalent under Condition 2 of the hypothesis (see Theorem 8, 9 and 10), Theorem 22 is a direct corollary to Theorem 21.

THEOREM 23. If $F_{1}, F_{2}, \cdots, F_{n}$ is a finite collection of disjoint closed subsets of a 2-sphere $S$ in $E^{3}$ such that either $\left(A, F_{i}, S\right)$ or $\left(B, F_{i}, S\right)$ is satisfied for each $i$, then $\left(A, \sum F_{i}, S\right)$ is satisfied.

Proof. We will show that $\left(A, F_{1}+F_{2}, S\right)$ is satisfied, then by induction and Theorem 9 the theorem will follow. Let $\varepsilon$ be a positive number. We assume that $3 \varepsilon<\rho\left(F_{1}, F_{2}\right)$. There are positive numbers $\delta_{1}$ and $\delta_{2}$, obtained using the definitions of $\left(A, F_{1}, S\right)$ and $\left(A, F_{2}, S\right)$, respectively, such that for each $i(i=1,2)$ each $\delta_{i}$-simple closed curve in $E^{3}-S$ can be shrunk to a point in an $\varepsilon$-subset of $E^{3}-F_{i}$. We choose $\delta=\min \left(\delta_{1}, \delta_{2}\right)$, and suppose that $J$ is a $\delta$-simple closed curve in $E^{3}-S$. Let $N$ be an $\varepsilon$-neighborhood of $J$. Since $\delta<\varepsilon$ it follows that $N$ is a $3 \varepsilon$-subset of $E^{3}$. Then $N$ cannot intersect both $F_{1}$ and $F_{2}$. Since $\delta \leqq \delta_{i}(i=1,2), J$ can be shrunk to a point in $N-\left(F_{1}+F_{2}\right)$. Thus we have established Property $\left(A, F_{1}+F_{2}, S\right)$.

REMARK. We do not know whether Theorem 23 is true without the requirement that the $F_{i}^{\prime}$ s be disjoint. We have an affirmative answer in the special case where the diameters of the components of each $F_{i}$ are bounded below by a positive number, since in this case Properties $\left(A, F_{i}, S\right), \quad\left(B, F_{i}, S\right)$, and $\left(*, F_{i}, S\right)$ are equivalent (see Theorems $8,9,10$, and 22 ).

\section{REFERENCES}

1. R. H. Bing, Locally tame sets are tame, Ann. of Math. 59 (1954), 145-158.

2. - Approximating surfaces with polyhedral ones, Ann. of Math. 65 (1957), 456-483.

3. - Conditions under which a surface in $E^{3}$ is tame, Fund. Math. 47 (1959), 105-139.

4. - A surface is tame if its complement is 1-ULC, Trans. Amer. Math. Soc. 101 (1961), 294-305.

5. - - A wild surface each of whose arcs is tame, Duke Math. J. 28 (1961), 1-15.

6. - Each disk in $E^{3}$ contains a tame arc, Amer. J. Math. 84 (1962), 583-590.

7. - Each disk in $E^{3}$ is pierced by a tame arc, Amer. J. Math. 84 (1962), 591-599.

8. - Approximating surfaces from the side, Ann. of Math. 77 (1963), 145-192. 
9. $33-45$.

10. C. E. Burgess, Characterizations of tame surfaces in $E^{3}$, Trans. Amer. Math. Soc. 114 (1965), 80-97.

11. P. H. Doyle and J. G. Hocking, Some results on tame disks and spheres in $E^{3}$, Proc. Amer. Math. Soc. 11 (1960), 832-836.

12. R. H. Fox and E. Artin, Some wild cells and spheres in three-dimensional space, Ann. of Math. 49 (1948), 979-990.

13. David S. Gillman, Side approximation, missing an arc, Amer. J. Math. 85 (1963), $459 \sim 76$.

14. Norman Hosay, Some sufficient conditions for a continuum on a 2-sphere to lie on a tame 2-sphere, Notices Amer. Math. Soc. 11 (1964), 370-371.

15. Witold Hurewicz and Henry Wallman, Dimension theory, Princeton University Press, 1948.

16. L. D. Loveland, Tame surfaces and tame subsets of spheres in $E^{3}$, Trans. Amer. Math. Soc. 123 (1966), 355-368.

17. E. E. Moise, Affine structures in 3-manifolds, VIII; Invariance of knot-types: Local tame imbeddings, Ann. of Math. 59 (1954), 159-170.

18. R. L. Moore, Concerning upper semicontinuous collections of continua, Trans. Amer. Math. Soc. 27 (1925), 416-428.

19. G. T. Whyburn, Topological characterization of the Sierpinski curve, Fund. Math. 45 (1958), 320-324.

Received July 26, 1965. This paper formed part of the author's doctoral thesis at the University of Utah under the direction of Professor C. E. Burgess. This research was supported by a National Aeronautics and Space Administration Fellowship.

The UNIVERSity of UTAH, Utah State University 



\section{PACIFIC JOURNAL OF MATHEMATICS}

\section{EDITORS}

H. SAMELSON

Stanford University

Stanford, California

J. P. JANS

University of Washington

Seattle, Washington 98105

\section{J. DuGUNDJI}

University of Southern California

Los Angeles, California 90007

RICHARD ARENS

University of California

Los Angeles, California 90024

\section{ASSOCIATE EDITORS}

E. F. BECKENBACH
B. H. NEUMANN

F. WOLF

K. YoSIDA

\section{SUPPORTING INSTITUTIONS}

\author{
UNIVERSITY OF BRITISH COLUMBIA \\ CALIFORNIA INSTITUTE OF TECHNOLOGY \\ UNIVERSITY OF CALIFORNIA \\ MONTANA STATE UNIVERSITY \\ UNIVERSITY OF NEVADA \\ NEW MEXICO STATE UNIVERSITY \\ OREGON STATE UNIVERSITY \\ UNIVERSITY OF OREGON \\ OSAKA UNIVERSITY \\ UNIVERSITY OF SOUTHERN CALIFORNIA
}

\author{
STANFORD UNIVERSITY \\ UNIVERSITY OF TOKYO \\ UNIVERSITY OF UTAH \\ WASHINGTON STATE UNIVERSITY \\ UNIVERSITY OF WASHINGTON \\ AMERICAN MATHEMATICAL SOCIETY \\ CHEVRON RESEARCH CORPORATION \\ TRW SYSTEMS \\ NAVAL ORDNANCE TEST STATION
}

Mathematical papers intended for publication in the Pacific Journal of Mathematics should be typewritten (double spaced). The first paragraph or two must be capable of being used separately as a synopsis of the entire paper. It should not contain references to the bibliography. Manuscripts may be sent to any one of the four editors. All other communications to the editors should be addressed to the managing editor, Richard Arens at the University of California, Los Angeles, California 90024 .

50 reprints per author of each article are furnished free of charge; additional copies may be obtained at cost in multiples of 50 .

The Pacific Journal of Mathematics is published monthly. Effective with Volume 16 the price per volume (3 numbers) is $\$ 8.00$; single issues, $\$ 3.00$. Special price for current issues to individual faculty members of supporting institutions and to individual members of the American Mathematical Society: $\$ 4.00$ per volume; single issues $\$ 1.50$. Back numbers are available.

Subscriptions, orders for back numbers, and changes of address should be sent to Pacific Journal of Mathematics, 103 Highland Boulevard, Berkeley 8, California.

Printed at Kokusai Bunken Insatsusha (International Academic Printing Co., Ltd.), No. 6, 2-chome, Fujimi-cho, Chiyoda-ku, Tokyo, Japan.

\section{PUBLISHED BY PACIFIC JOURNAL OF MATHEMATICS, A NON-PROFIT CORPORATION}

The Supporting Institutions listed above contribute to the cost of publication of this Journal, but they are not owners or publishers and have no responsibility for its content or policies. 


\section{Pacific Journal of Mathematics

Vol. 19, No. $3 \quad$ July, 1966

S. J. Bernau, The spectral theorem for unbounded normal operators .......

Lu-san Chen, Asymptotic behavior of solutions of parabolic equations of

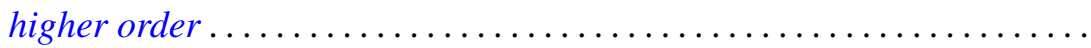

Lawrence William Conlon, An application of the Bott suspension map to the

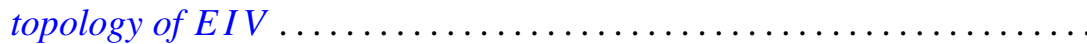

Neal Eugene Foland and John M. Marr, Sets with zero-dimensional kernels .........................................

Stanley Phillip Franklin and R. H. Sorgenfrey, Closed and image-closed

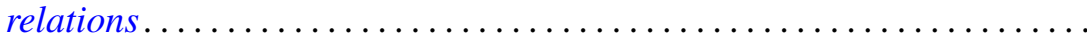

William Jesse Gray, A note on topological transformation groups with a

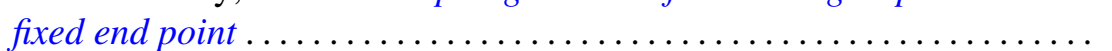

Myron Goldstein, $K$ - and L-kernels on an arbitrary Riemann surface ...... 449

George Joseph Kertz and Francis Regan, The exponential analogue of a generalized Weierstrass series .............................

Walter Leighton, On Liapunov functions with a single critical point ........ 467

Bernard Werner Levinger and Richard Steven Varga, On a problem of $O$.

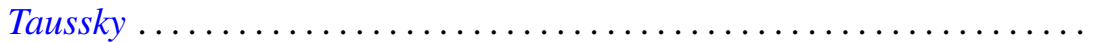

Lowell Duane Loveland, Tame subsets of spheres in $E^{3} \ldots \ldots \ldots \ldots \ldots . .489$

Erik Andrew Schreiner, Modular pairs in orthomodular lattices ......... 519

K. N. Srivastava, On dual series relations involving Laguerre polynomials ...............................

Arthur Steger, Diagonability of idempotent matrices.....

Walter Strauss, On continuity of functions with values in various Banach spaces...

Robert Vermes, On the zeros of a linear combination of polynomials ...

Elliot Carl Weinberg, On the scarcity of lattice-ordered matrix rings ....

Harold Widom, Toeplitz operators on $H_{p} \ldots \ldots \ldots \ldots \ldots$

Neal Zierler, On the lattice of closed subspaces of Hilbert space...

Irving Leonard Glicksberg, Correction to: "Maximal algebras and a theorem of Rado"

John Spurgeon Bradley, Correction to: "Adjoint quasi-differential operators of Euler type"

William Branham Jones, Erratum: "Duality and types of completeness in locally covex spaces".

Stanley P. Gudder, Erratum: "Uniqueness and existence properties of bounded observables" 